\title{
Temporary Inactivation of the Retrosplenial Cortex Causes a Transient Reorganization of Spatial Coding in the Hippocampus
}

\author{
Brenton G. Cooper and Sheri J. Y. Mizumori \\ Department of Psychology, University of Utah, Salt Lake City, Utah 84112
}

The ability to navigate accurately is dependent on the integration of visual and movement-related cues. Navigation based on metrics derived from movement is referred to as path integration. Recent theories of navigation have suggested that posterior cortical areas, the retrosplenial and posterior parietal cortex, are involved in path integration during navigation. In support of this hypothesis, we have found previously that temporary inactivation of retrosplenial cortex results in darkselective impairments on the radial maze (Cooper and Mizumori, 1999). To understand further the role of the retrosplenial cortex in navigation, we combined temporary inactivation of retrosplenial cortex with recording of complex spike cells in the hippocampus. Thus, behavioral performance during spatial memory testing could be compared with place-field responses before, and during, inactivation of retrosplenial cortex. In the first experiment, behavioral results confirmed that inactivation of retrosplenial cortex only impairs radial maze performance in darkness when animals are at asymptote levels of performance. A second experiment revealed that retrosplenial cortex inactivation impaired spatial learning during initial light training. In both experiments, the normal location of hippocampal "place fields" was changed by temporary inactivation of retrosplenial cortex, whereas other electrophysiological properties of the cells were not affected. The changes in place coding occurred in the presence, and absence, of behavioral impairments. We suggest that the retrosplenial cortex provides mnemonic spatial information for updating location codes in the hippocampus, thereby facilitating accurate path integration. In this way, the retrosplenial cortex and hippocampus may be part of an interactive neural system that mediates navigation.

Key words: navigation; place cells; path integration; spatial memory; head direction; posterior cingulate cortex
Navigation requires the continual updating of one's current location based on stable visual features of the environment and movement through space (Gallistel, 1990; Etienne, 1992). Understanding the neural basis of navigation requires, at the very least, identification of the different structures involved in integrating visual cues with movement during navigation.

Hippocampal pyramidal cells fire robustly when animals traverse particular locations in space; these locations are called the "place field" of the cell (O'Keefe and Dostrovsky, 1971). The contribution of visual cues to place fields has been illustrated by rotating the salient cue(s) in a testing arena (O'Keefe and Conway, 1978; Muller et al., 1991). Most place fields are bound to the distal cues, and simultaneously recorded neurons rotate synchronously suggesting that neuronal ensembles follow distal cues (O’Keefe and Speakman, 1987; Knierim et al., 1995). However, place fields can develop in blind rats (Hill and Best, 1981; Save et al., 1998), in the absence of visual cues (Quirk et al., 1990), and maintain spatial firing when visual cues are removed (Muller et al., 1991; Markus et al., 1994; Mizumori et al., 1999). Accordingly, there has been an increased interest in the role of movement-

\footnotetext{
Received May 10, 2000; revised Jan. 19, 2001; accepted March 12, 2001.

This work was supported by National Institutes of Health Predoctoral Fellowship MH 11998 to B.G.C. and Grant MH 58755 to S.J.Y.M. We thank Gena Ettinger and Theodore F. Manka for help in behavioral training, Wayne Pratt and Alex Guazzelli for helpful comments on this manuscript, James Canfield for extraordinary technical assistance on a variety of aspects of this project, and Stefan Leutgeb and Alex Guazzelli for outstanding programming assistance.

Correspondence should be addressed to Dr. Sheri J. Y. Mizumori, Department of Psychology, Box 351525, University of Washington, Seattle, WA 98195-1525. E-mail: mizumori@u.washington.edu.

B. G. Cooper's present address: Department of Biology, University of Utah, Salt Lake City, UT 84112.

Copyright (C) 2001 Society for Neuroscience $\quad 0270-6474 / 01 / 213986-16 \$ 15.00 / 0$
}

related cues in hippocampal spatial processing. Manipulations designed to disrupt or manipulate self-motion cues can disrupt or systematically alter place fields of hippocampal neurons (Sharp et al., 1990, 1995; Knierim et al., 1995; Gothard et al., 1996; Jeffery et al., 1997; Jeffery and O'Keefe, 1999).

McNaughton et al. (1996) suggest that posterior parietal and retrosplenial cortex may send self-motion information to the hippocampus. On the basis initially of anatomical observations, we further hypothesized that retrosplenial cortex facilitates the experience-dependent integration of visual and self-motion cues. Visual projections from both the geniculostriate and tectocortical pathways converge in retrosplenial cortex (van Groen and Wyss, 1990a; Zilles and Wree, 1995). Information related to one's movement may arrive in retrosplenial cortex via direct projections between posterior parietal cortex and anterior thalamic nuclei (ATN) (van Groen and Wyss, 1995; Zilles and Wree, 1995). A subset of cells in retrosplenial cortex is sensitive to the direction an animal is facing in an environment; these cells are commonly referred to as "head-direction cells." Retrosplenial cortex head-direction cells are modulated by visual and movement cues (Chen et al., 1994a,b). In agreement with our initial hypothesis, temporary inactivation of retrosplenial cortex causes behavioral impairments when animals perform a well learned spatial memory task in darkness but does not disrupt performance when visual cues are available (Cooper and Mizumori, 1999).

To understand better the contribution of retrosplenial cortex to navigation and hippocampal spatial processing, temporary inactivation of retrosplenial cortex was combined with recording of hippocampal cells in well trained rats during light and dark maze performance. We predicted that place cells would change their 
spatial coding during inactivation, especially when behavioral impairments were evident. In a second experiment, retrosplenial cortex was inactivated before the animals initially learned the spatial memory task in light conditions. If retrosplenial cortex contributes to mnemonic integration of visual and self-motion information, we predicted that inactivation would cause spatiallearning impairments and place-field instability during initial visual spatial learning.

\section{MATERIALS AND METHODS}

Subjects. Eleven male Long-Evans rats obtained from Simonson's Laboratories (Gilroy, CA) were used in the experiments. Animals were kept in a temperature- and humidity-controlled room with a $12 \mathrm{hr}$ light cycle (lights on at 7:00 A.M.). One week was given to allow the rats to acclimate to the laboratory before onset of experimental procedures. During this time animals were handled and weighed daily. During behavioral testing and training, animals were food restricted and maintained at $80 \%$ of their free-feeding weights. Animal testing was conducted in an Association for Accreditation of Laboratory Animal Careapproved facility, within the guidelines of National Institutes of Health animal care and use.

Behavioral method. Animals were trained to perform a spatial memory task on an eight-arm radial maze using procedures similar to those described elsewhere (Mizumori et al., 1989; Cooper et al., 1998; Cooper and Mizumori, 1999). Briefly, the maze is elevated $(77 \mathrm{~cm})$ above the floor and consists of eight arms $(59.5 \times 5 \mathrm{~cm})$ with $0.5 \mathrm{~cm}$ railings radiating from a center platform (19.5 cm in diameter). Arm access is afforded or restricted via remote control by raising or lowering, respectively, the proximal portion of the maze arm. A camera positioned above the maze allowed the experimenter to monitor the animals from an adjacent room. In darkness, movement of the animal was monitored by observing a pair of infrared diode arrays attached to the head of the rat. Two mazes with identical physical dimensions were located in two different testing rooms. One room was used solely for behavioral training (room 1). Room 1 was a large open room containing numerous distal cues surrounding the maze. The maze for electrophysiological recording was enclosed in a controlled cue environment consisting of black curtains forming a square around the maze (room 2). A canopy-style ceiling started at the camera and draped down to the four walls of the enclosure. The room contained numerous distal cues for use by the animals to determine their location and directional heading within the environment [for further description of room 2, see Cooper et al. (1998)].

In room 1, animals were habituated to the maze and then trained to retrieve chocolate milk from the end of randomly selected maze arms. On each trial, the experimenter randomly selected eight maze arms, which were individually presented until the animal had visited all maze arms. After this "forced-choice" nonspatial memory training, animals were then trained to perform a "win-shift" spatial memory task in room 2. The spatial memory task consisted of two phases. In the first phase animals were presented with four randomly selected arms individually and sequentially. While animals were on the fourth arm, the second phase began by raising all eight maze arms. Animals were allowed to chose freely among the eight arms; arm reentries were considered errors. This partial forced-choice procedure minimizes the possibility that animals will develop a response-based strategy for solving the task.

Electrode construction and surgical procedure. Hippocampal single-unit activity was recorded using the stereotrode recording technique (McNaughton et al., 1983b). Two lacquer-coated tungsten wires $(25 \mu \mathrm{m}$; California FineWire) were twisted together, dipped in Epoxylite, and baked. The stereotrode was then threaded through a 30 ga stainless steel tube, and two to four cannulas were mounted on a moveable microdrive; two microdrives were implanted per animal.

Similar to procedures used previously, guide cannulas were cut from 25 ga stainless steel tubing at a length of $1.2 \mathrm{~cm}$ (Mizumori et al., 1989, 1990, 1994; Cooper and Mizumori, 1999). Removable stylets were constructed from 33 ga stainless steel tubing and were placed inside of the guide cannulas to prevent occlusion of the tubing. By the use of a stereotaxic drill assembly, holes were drilled in $0.15 \mathrm{~mm}$ nylon sheeting, and guide cannulas were glued in the holes $2 \mathrm{~mm}$ apart from each other. Injection needles were made of 33 ga stainless steel tubing glued inside of 25 ga stainless steel tubing. The injection needles protruded $\sim 0.5 \mathrm{~mm}$ beyond the guide cannulas. Stylets remained in the guide cannulas after surgery and between injections.
Animals were food and water deprived for $24 \mathrm{hr}$ before surgery and then anesthetized with sodium pentobarbital $(33 \mathrm{mg} / \mathrm{kg})$. After animals were deeply anesthetized, they were given $0.2 \mathrm{ml}$ of Atropine to prevent respiratory distress. Ten burr holes were drilled, and self-tapping anchor screws were inserted into the holes. Hippocampal electrodes were placed in the dorsal hippocampus at two recording sites. Recordings from the right hippocampus were at $1.8-2.2 \mathrm{~mm}$ posterior to bregma and $1.8 \mathrm{~mm}$ lateral. In the left hemisphere, electrodes were placed $2.5-4.0 \mathrm{~mm}$ posterior and $2.0-2.5 \mathrm{~mm}$ lateral. Dura was cut, and electrodes were lowered $1.5 \mathrm{~mm}$ ventral to the surface of the brain, just dorsal to the hippocampus. The right and left hemisphere placements were selected to maximize the amount of room available around the guide cannulas, providing easy access for insertion of the injection needles. Retrosplenial cortex craniotomies were drilled at $6.0 \mathrm{~mm}$ posterior to bregma and 1.0 $\mathrm{mm}$ lateral to the midline. Dura was cut, and guide cannulas were implanted $1.0 \mathrm{~mm}$ ventral to the surface of the brain. A reference electrode (114 $\mu \mathrm{m}$ Teflon-coated stainless steel wire) was placed near the corpus callosum, and a ground lead (125 $\mu \mathrm{m}$ Teflon-coated stainless steel wire) was soldered to a stainless steel jeweler's screw that was fastened to the skull. Vacuum grease was packed around the electrodes and guide cannulas to protect the surface of the brain from exposure to dental cement. The microdrives and guide cannulas were permanently attached to the head of the rat by application of dental cement. The electrode wires were connected to an 18-pin plug that was cemented behind the microdrives and guide cannulas. After surgery, $0.1 \mathrm{ml}$ of Bicillin $(300,000$ $\mathrm{U} / \mathrm{ml}$ ) was administered intramuscularly in each hindlimb to guard against infection. Animals were given 1 week of free feeding before the onset of food restriction and experimental procedures.

Unit recording and behavioral monitoring. The rat was connected to a head stage for all recording sessions. The head stage contained 13-16 field effect transistors and a pair of infrared-emitting diode arrays. The location of the animal on the maze was monitored via an automatic tracking system (Dragon Tracker, Boulder, CO) that sampled position data at a frequency of $20 \mathrm{~Hz}$. The tracking system identified two diode arrays simultaneously, distinguished them on the basis of size of the array, and gave $x-y$ coordinates in a $256 \times 256$ grid for each array. The front array was placed directly over the nose of the animal. It was comprised of five to seven infrared diodes that identified the location of the animal. A smaller rear diode array (made of one or two diodes) was placed $12 \mathrm{~cm}$ behind the front array. The pair of diode arrays was used to calculate the heading direction of the animal.

Single-unit activity was recorded simultaneously and independently on each wire of the stereotrode pair. Each signal was amplified $(3,000-$ $10,000 \times)$, filtered at half amplitude between 600 and $6 \mathrm{kHz}$, and then passed through a window discriminator such that a $1 \mathrm{msec}$ sampling period began when either input surpassed a predetermined threshold. The DataWave "Discovery" data acquisition system recorded each ana$\log$ trace at a frequency of $20-32 \mathrm{kHz}$ depending on the number of simultaneously recorded electrodes. The system software allowed the experimenter to isolate individual units from the otherwise multiunit record by comparing the spike characteristics recorded simultaneously on two closely spaced electrodes $(x$ and $y)$. Scatterplots of waveform features recorded on $x$ and $y$ electrodes were displayed. Multiple waveform parameters were used to separate individual cells from each other and from background noise. Particularly useful features included spike amplitude, spike width (time differences between the peak and subsequent trough of an action potential signal), and the latency differences between the spike peak and valley on the $x$ and the $y$ electrodes. In addition, a template-matching algorithm was used off-line to facilitate spike separation further. For each cell, the experimenter stepped through a series of two-dimensional cluster plots identifying the combination of spike characteristics that were most likely associated with a single-spike generator. After being identified, the specific cluster boundaries that characterized each cell were saved for use in subsequent recording sessions. This provided reasonable certainty that the same cell was being recorded across multiple test days.

\section{Experiment 1}

The present experiment was intended to replicate and extend our previous behavioral findings of a dark-selective spatial memory impairment after retrosplenial cortex inactivation (Cooper and Mizumori, 1999). We sought to extend those results by addressing potential neural mechanisms underlying dark spatial memory impairments. Therefore, we examined changes in hippocampal cellular activity during retrosplenial cortex inactivation in light and dark testing conditions. 
A.

Light Inactivation

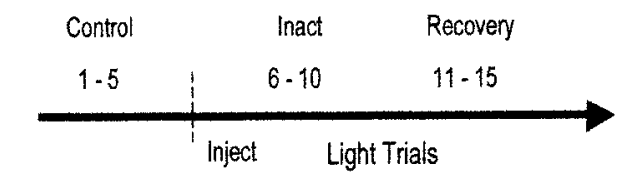

B.

Dark I Inactivation

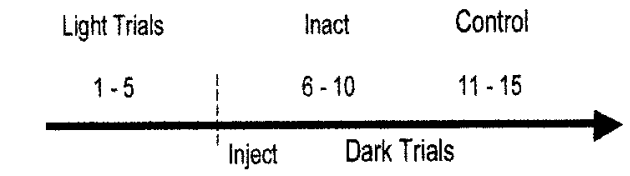

C.

Dark II Inactivation

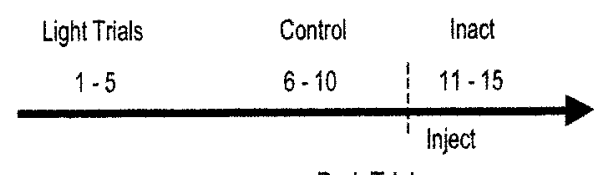

Dark Trials

Figure 1. The injection procedure used for light inactivation and dark I and dark II inactivation. A dashed vertical line and Inject denote the time of injection; Inact indicates the trials used for the inactivation condition. $A$, In light inactivation, the first five trials serve as control trials for comparison with trials $6-10$ that are inactivation trials. The final set of five trials was used to ensure that behavioral and electrophysiological changes returned toward control levels. $B$, In dark I inactivation, the injection occurred before the onset of dark trials. $C$, In dark II inactivation, the injection took place after the first five dark trials.

Behavioral and injection methods. After 2 weeks of spatial memory training (in room 2) and error rates had decreased to an average of less than one error per trial, animals were considered to be at asymptote performance. At this point animals were run every third day unless hippocampal unit activity was encountered. When well isolated units were located, animals performed the spatial memory task in light and dark testing conditions with or without the retrosplenial cortex temporarily inactivated.

Previous work has demonstrated that tetracaine $(2 \%)$ is active for $\sim 20$ min, which is the equivalent of approximately five maze trials (Mizumori et al., 1989, 1990, 1994; Cooper and Mizumori, 1999). Therefore, most of the behavioral and electrophysiological analyses are based on five control and five inactivation trials. All injections were accomplished by hand via a Hamilton syringe. Tetracaine was slowly infused over the course of 2-3 min, and 1 min was allowed for diffusion.

Figure 1 displays the experimental design used for inactivation during light and dark testing. For the light inactivation testing, animals first ran five control spatial memory trials. They were then removed from the maze and taken into an adjacent room, and $1 \mu l$ of tetracaine was inf used bilaterally into the retrosplenial cortex. Animals were then returned to the maze room and performed trials $6-10$ under normal lighting conditions. Trials $1-5$ were considered control trials and were compared with trials 6-10 (Fig. 1A). The final set of five trials was used to monitor the extent to which behavioral and electrophysiological changes returned toward control levels.

Consistent with our previous work, two versions of the dark injection procedure were used to control for the time of injection during dark trials (Cooper and Mizumori, 1999). For the dark I testing procedure, animals ran five maze trials in the light, were removed from the maze, and were taken into an adjacent room, and $1 \mu \mathrm{l}$ of tetracaine was infused bilaterally into the retrosplenial cortex. One minute was allowed for diff usion, and animals were returned to the maze in the light. Room lights were turned off immediately before the onset of testing, and animals performed 10 maze trials in darkness. For the dark I inactivation procedure, trials $6-10$ were dark inactivation trials, and trials $11-15$ were control dark trials (Fig. 1B). In the dark II inactivation procedure, animals ran five light trials, were removed from the maze, and were taken into an adjacent maze room; injection cannulas were inserted, but tetracaine was not infused at this time. Animals were returned to the maze in the light, room lights were extinguished, and then animals performed trials $6-10$ in darkness. After trial 10 (during dark trials), room lights were turned on, and animals were removed from the maze. Tetracaine $(1 \mu \mathrm{l})$ was inf used bilaterally into the retrosplenial cortex in an adjacent room, and animals were then returned to the maze to perform trials $11-15$ in darkness (inactivation trials). For the dark II procedure, trials 6-10 were control dark trials, and trials $11-15$ were dark inactivation trials (Fig. 1C). Regardless of which dark testing procedure was used, animals always ran five control and five inactivation dark trials. The control trials in darkness occurred either after tetracaine had worn off (i.e., Dark I) or before it was injected (i.e., Dark II). The two types of injection procedures were used to control for the potential confounds of strategy switching that may occur with initial, or prolonged, dark testing.

Behavioral and electrophysiological data analyses. The experimenter recorded the number of errors (i.e., repeat arm entries) and the time taken to complete each trial during the course of the behavioral testing. An average number of errors per trial during control and inactivation phases of testing was computed for each animal during light and dark testing. Time per choice was calculated by dividing the time to complete each trial by the number of arms visited within the trial. Changes in error rates and time per choice were evaluated by computing a two-way repeated measures ANOVA comparing light and dark trials with $\alpha=0.05$.

Hippocampal cells can be divided into pyramidal complex spike (CS) and interneuron single-spike $(\theta)$ cell populations (Fox and Ranck, 1975, 1981). These separate populations are readily identifiable on the basis of their unique spike characteristics. CS cells have broader spikes $(>300$ $\mu$ sec from peak to valley) than do $\theta$ cells and fire at a relatively low rate. In addition, CS cells show a characteristic bursting pattern of three to four action potentials occurring within $2-4 \mathrm{msec}$ of each other. $\theta$ Cell spikes occur $\sim 8 \mathrm{msec}$ apart, with a much higher overall firing rate than CS cells have, and they have a narrower spike width $(<300 \mu \mathrm{sec}$ from peak to valley). These spike characteristics are derived from stored analog traces of the waveforms, autocorrelations, and interspike interval histograms of the individual cells (Markus et al., 1994).

All spatial analyses of the electrophysiological data are based on reducing the $256 \times 256$ grid to a $64 \times 64$ grid, resulting in quadratic pixels $\cong 2.4 \times 2.4 \mathrm{~cm}$. Spatial specificity was quantified by first calculating the mean firing rate of the cell as the rat moved toward the center platform (inbound) or away from the center platform (outbound) on each of the eight maze arms yielding 16 average rates. The spatial specificity score was determined by dividing the highest of the 16 rates by the average of the remaining 15 (McNaughton et al., 1983a; Mizumori et al., 1989, 1992, 1994). Reliability was the proportion of trials that the cell fired maximally on the same arm and direction (inbound or outbound) across trials. In agreement with previous studies, cells with a spatial specificity score $\geq 2.5$ and a reliability of 0.40 were considered to have a place field. This indicates that a cell discharged at least 2.5 times its average rate as the rat moved on one arm in one direction for at least two of five trials. In addition to the spatial specificity score, information content and sparsity were calculated. Theoretically, information content is a measure of how well the firing rate of the cell predicts the location of an animal within an environment (Skaggs et al., 1993). Larger information content scores reflect smaller place fields. Information content is defined as:

$$
\text { information content }=\Sigma P_{j}\left(R_{j} / R\right) \log 2\left(R_{j} / R\right) .
$$

$P_{j}$ is the probability that a rat will occupy bin $j, R_{j}$ is the mean firing rate for bin $j$, and $R$ is the mean firing rate across the entire maze. Sparsity is a measure of the size of the place field and is defined as:

$$
\text { sparsity }=\left(\Sigma P_{j} R_{j}\right)^{2} / \Sigma P_{j} R_{j}{ }^{2} .
$$

$P_{j}$ is the probability that the rat occupied bin $j$, and $R_{j}$ is the mean firing rate for bin $j$.

The animal moving down the first arm identified the beginning of each trial, and the end was determined when the animal had visited each of the eight maze arms. The measures of spatial selectivity (spatial specificity, 

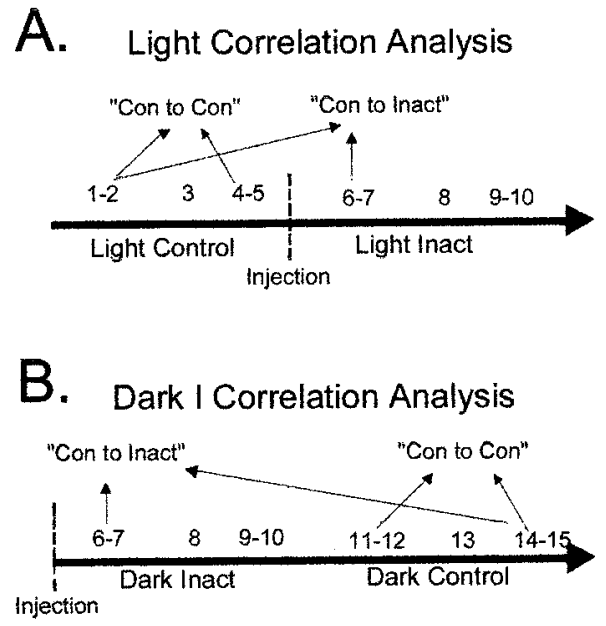

C. Dark II Correlation Analysis

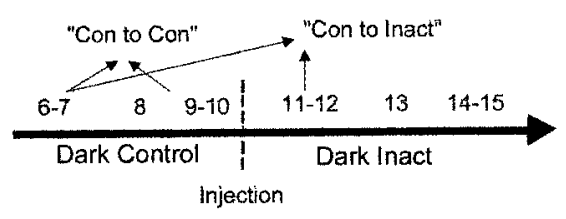

Figure 2. The trials used for the spatial correlation analysis during control and inactivation trials are displayed in the figure. The last five trials during light inactivation and the first five light trials for dark I and dark II inactivation are omitted from the spatial analysis; accordingly they are not included in the figure. $A$, For light inactivation, the firing rates on visited pixels during the first two trials of the control trials were correlated with the rates on visited pixels during the last two trials of the control condition (Con to Con). This provided the control stability assessment of place fields. To assess the effects of inactivation, the first two trials of the control trials were correlated with the first two inactivation trials (Con to Inact). $B$, The first two dark trials after inactivation were compared with the last two dark trials performed (Con to Inact) for the spatial correlation. Trials 11 and 12 were compared with trials 14 and 15 for the Con to Con spatial correlation. $C$, For dark II inactivation, the first two dark trials $(6,7)$ were compared with trials 9 and 10 for the Con to Con spatial correlation. The Con to Inact spatial correlation was derived from comparing trials 6 and 7 with the first two trials after inactivation (trials 11, 12).

information content, etc.) were calculated for each trial, and then means were determined for the five control, five inactivation, and five recovery trials.

If the place field of a cell changes location relative to a baseline sampling period and remains stable for several trials, the measures of spatially localized firing described above might not show significant differences across phases of testing. Accordingly, a spatial correlation was computed that examined the extent to which firing-rate maps were correlated within and across control and inactivation trials. Figure 2 displays the trials from the light inactivation (Fig. $2 A$ ), dark I inactivation (Fig. 2B), and dark II inactivation (Fig. $2 C$ ) that were used for the spatial correlation analysis. The analysis was based on a pixel-by-pixel correlation of average firing rates during the first two trials of the control condition compared with the average of the last two trials of the control condition (Fig. 2, "Con to Con"). To examine the effects of retrosplenial cortex inactivation on the place fields, the spatial correlation was computed comparing the average rates of the first two trials of the control condition and the first two inactivation trials (Fig. 2, "Con to Inact"). If the spatial distribution of a place field is similar during control and inactivation trials, the correlation between con to con and con to inact should be comparable. In this case, the place field would be considered stable across the conditions. If the place field changes location, enlarges in size, or reduces in size or the firing rate of the cell changes, then the con-to-con correlation would be higher than the con-to-inact correlation. In this case, the field would be considered unstable across conditions. A within-subjects $t$ test was used to determine whether the CS place-field correlation changed significantly from the control to inactivation conditions.

Two phases of testing were analyzed with the spatial correlation, a control phase was compared with another control phase, or a control phase was compared with an inactivation phase. The spatial correlation only included bins that were visited in both phases for at least $200 \mathrm{msec}$. The joint occupancy requirement ensured that only areas of the maze that were sampled consistently across phases of testing were used in the correlation analysis. The use of two trial segments from each testing phase was chosen for two reasons. First, previous research has shown that the most reliable and pronounced electrophysiological effects of inactivation in freely behaving animals occur during the first two trials after injection (Mizumori et al., 1989, 1994). Last, the two trial segments provided the largest number of trials that allowed each phase (control and inactivation) to be divided into an equal number of trials for assessing place-field stability within and across phases of testing. The spatial correlation used in the present study is similar to analyses used in other reports (Knierim et al., 1995, 1998; Barnes et al., 1997). However, the absolute numbers may vary substantially across experiments depending on the amount of time sampled for the correlation and the size of the pixels (binning resolution). Therefore, relative changes in the spatial correlation are appropriate for comparison with other studies.

\section{Experiment 2}

Behavior and injection procedure. Six animals were first trained to retrieve chocolate milk from the end of randomly presented maze arms in room 1 using a forced-choice nonspatial task (see Behavioral method in Materials and Methods). During this initial training, animals were checked daily for hippocampal single-unit activity. After multiple complex spike cells were identified and animals were running consistently on the maze in room 1 , rats were randomly assigned to either tetracaine $(n=3)$ or control $(n=3)$ groups. The tetracaine group received daily injections (1 $\mu \mathrm{l} /$ hemisphere) before the onset of spatial memory training in room 2 . Similarly, the control group received vehicle control injections $(1 \mu \mathrm{l} /$ hemisphere) before the onset of spatial memory training in room 2. After the injection of tetracaine or the vehicle control, 1 min was allowed for diffusion, and animals were immediately carried into the testing room. Spatial memory trials commenced as quickly as possible after the injection. Animals performed five trials or ran maze trials for a maximum of $20 \mathrm{~min}$, whichever occurred first. Training continued for 5 consecutive days, while hippocampal unit activity was recorded. Efforts were made to identify carefully the same cells across days during the acquisition, but not all cells could be recorded for 5 continuous days.

Behavioral and electrophysiological data analyses. Behavioral data were analyzed by computing the mean number of errors committed on each trial during each test day. A mixed-design ANOVA comparing average errors between the tetracaine and saline groups across days $(\alpha=0.05)$ was used to analyze the acquisition data. Electrophysiological measures were also analyzed using a mixed-design ANOVA. The analysis requires that each "subject" contributes a score for each day. Some cells were recorded across days, whereas new cells were also encountered on different days. Thus, a single cell could not always contribute a score for each day in the repeated measures component of the ANOVA. To resolve this issue, a mean score for each animal based on the cells recorded from that day was used in the analysis. Mean spatial specificity, reliability, information content, sparsity, mean rate, and the spatial correlation measure were calculated for each day during acquisition. Two criteria were necessary to calculate the spatial correlation score. First, each animal had to run at least four trials on each day of acquisition of the spatial memory task. In this case, the first two trials were correlated with the last two trials. When animals ran five trials during acquisition training, trials 1 and 2 were compared with trials 4 and 5; the majority of the analyses are based on this comparison. Second, at least one CS cell had to be recorded from each animal during each day of the spatial memory task. These criteria were met on days $2-4$ of spatial memory acquisition. Therefore, the statistical analyses for all measures of spatial coding by hippocampal neurons were restricted to these days. However, the behavioral analysis compares all $5 \mathrm{~d}$ of acquisition. 
A.

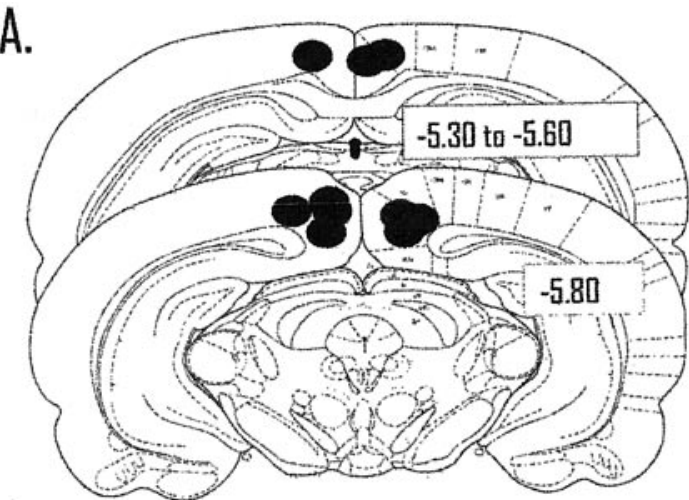

B.

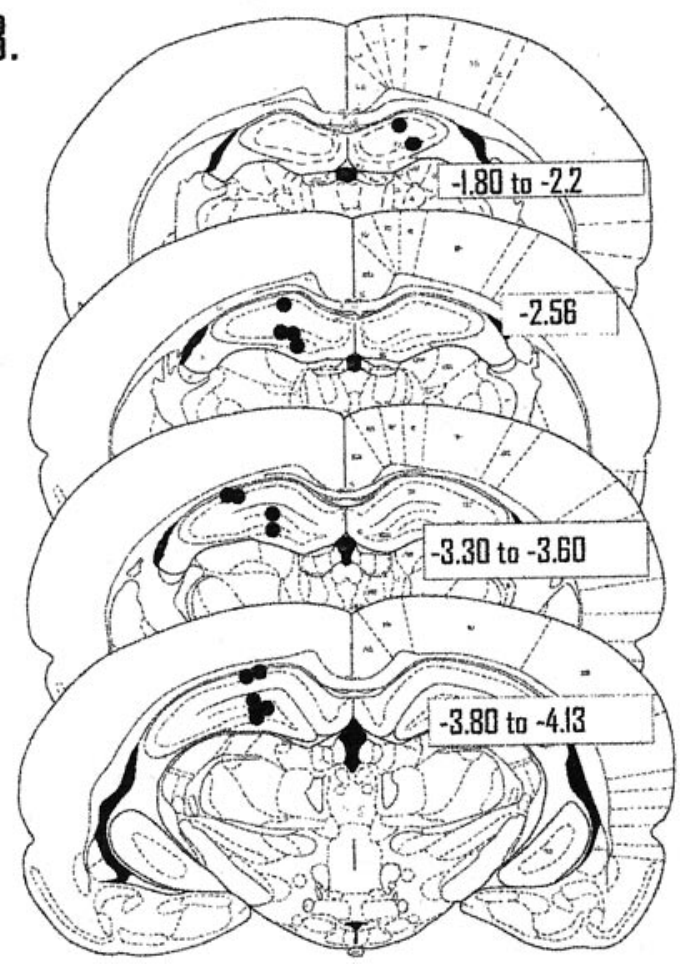

Figure 3. Location of the tips and recording sites of the guide cannulas. $A$, Each filled circle corresponds to a single guide cannula. Previous work and ink injections have shown that the spread of tetracaine is just slightly more than a $1 \mathrm{~mm}$ circumference around the injection site. Therefore, retrosplenial granular and agranular areas, cingulum bundle, and Oc2MM of posterior parietal cortex were likely affected by injections of tetracaine. $B$, Each filled circle corresponds to two to eight cells recorded in that location. For electrode tracks that passed through the same area in different animals, a single filled circle is used to signify the recording site of multiple cells. The majority of cells $(n=43)$ were recorded from CA3 in the left hemisphere; a smaller number of cells $(n=15)$ were also recorded in CA1.

\section{RESULTS}

\section{Experiment 1}

Histology

Figure $3 A$ displays the locations of the tips of the cannulas in the retrosplenial cortex from six animals (Paxinos and Watson, 1986). Three other animals were excluded from the behavioral and most of the electrophysiological analyses because of the placements of the cannulas. In one animal the tip of the cannula was placed in the superior colliculus. This animal did not contribute control injection data and was excluded from all behavioral and electro- physiological analyses. In two other animals, only one of the two cannulas penetrated the cortex. The animals with unilateral guide cannulas were excluded from behavioral and electrophysiological analyses of retrosplenial cortex inactivation, but their data were kept for all control injections. In several cases, permanent ink was injected before killing the rat. In these animals, ink spread into the retrosplenial granular and agranular areas, cingulum bundle, and medial areas of posterior parietal cortex (Oc2MM), consistent with our previous work (Cooper and Mizumori, 1999). The ink did not spread into any subregions of the hippocampus.

The recording sites in the hippocampus from the six animals are displayed in Figure $3 B$. Each filled circle corresponds to approximate recording sites of two to eight cells. Electrode tracks that passed through similar areas across animals were grouped together for the graphical display of recording sites. Most of the recordings were from the left hemisphere in hilar/CA3 and to a lesser extent CA1. The recording sites were evenly distributed across animals, with the exception that one animal contributed the cells recorded in the right hemisphere. A total of $10 \mathrm{CS}$ cells and $1 \theta$ cell were recorded in the right hemisphere. In the left hemisphere, $12 \mathrm{CS}$ cells and $3 \theta$ cells were recorded in CA1, and $36 \mathrm{CS}$ cells and $7 \theta$ cells were recorded in CA3 during inactivation of retrosplenial cortex. The larger number of cells in CA3 was an inadvertent consequence of the fact that some of the animals took part in experiment 1 after completing experiment 2 (described below). Therefore, some of the recording electrodes passed through CA1 before commencing the current experiment. Retrosplenial cortex inactivation did not result in obvious differences in electrophysiological changes between hippocampal subregions or across animals. Therefore, the data were combined for the electrophysiological analyses.

\section{Behavioral data}

Experiment 1 replicated our previous behavioral data (Cooper and Mizumori, 1999). A two-way repeated measures ANOVA demonstrated that inactivation of retrosplenial cortex resulted in a significant increase in errors when retrosplenial cortex was inactivated and animals were tested during dark spatial memory performance. There were significant main effects of injection condition $\left[F_{(1,8)}=14.74 ; p<0.01\right]$ and lighting condition $\left[F_{(1,1)}\right.$ $=12.04 ; p<0.02]$. The dark-specific changes in behavioral performance caused by inactivation of retrosplenial cortex are illustrated by the significant interaction between lighting condition and trials during the injection condition $\left[F_{(1,4)}=4.30 ; p<\right.$ $0.05]$. Figure $4 A$ displays the absence of a change in error rates when retrosplenial cortex was inactivated during light testing. Figure $4 C$ shows the significant increase in error rates during dark testing when retrosplenial cortex was inactivated. The darkspecific behavioral deficit did not depend on the time of injection during dark trials (data not shown).

Figure $4, B$ and $D$, displays the average time per choice across trials in light and dark testing conditions. A two-way repeated measures ANOVA did not show main effects for lighting condition $\left[F_{(1,8)}=0.16 ; \mathrm{NS}\right]$ or injection condition $\left[F_{(1,1)}=0.75 ; \mathrm{NS}\right]$, which demonstrates that maze run times did not change with either dark testing or inactivation of retrosplenial cortex. One animal did not undergo inactivation while recording hippocampal cells but contributed to control injection data; therefore the statistical analyses are based on five of the six animals with bilateral guide cannulas placed in the retrosplenial cortex. 

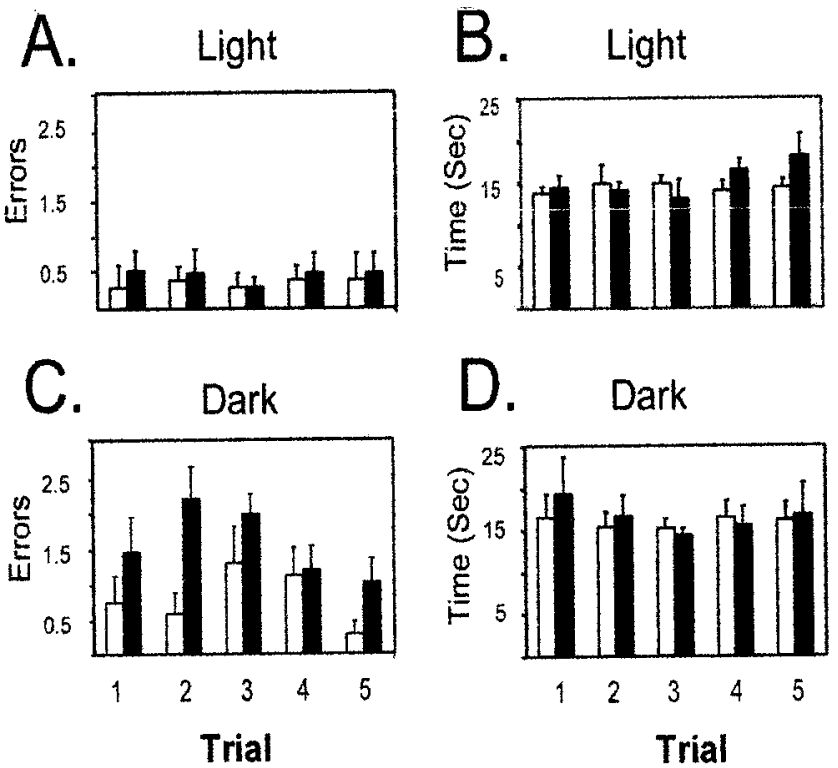

Figure 4. Temporary inactivation of retrosplenial cortex only impairs dark spatial memory performance. $A, C$, The average number of errors $( \pm$ SEM) for control and inactivation trials is displayed. Inactivation of retrosplenial cortex did not change performance during light testing $(A)$ but caused a significant impairment when animals were tested in darkness with retrosplenial cortex inactivated $(C) . B, D$, The average time per choice on the maze was not affected by retrosplenial cortex inactivation during light $(B)$ or dark $(D)$ inactivation. Because the data obtained from dark I and dark II were similar, they were combined for the present analysis.

\section{Inactivation effects on $\theta$ cells in light and dark}

Seven $\theta$ cells were recorded during inactivation of retrosplenial cortex in light testing conditions, and there were no significant changes in reliability, information content, or selectivity (Table $1)$. The average firing rate of $\theta$ cells increased from 13.70 ( \pm 1.98$)$ $\mathrm{Hz}$ during control light trials to $15.02( \pm 2.17) \mathrm{Hz}$ during light inactivation trials $[t(6)=-3.04 ; p<0.05$; Table 1]. Eight $\theta$ cells were tested with inactivation of retrosplenial cortex in darkness. There were no significant differences between injections in dark I or dark II; therefore the data were combined for the statistical analyses. There were no significant changes in any of the measures of spatial coding or mean rate when retrosplenial cortex was inactivated during dark trials (Table 1).

\section{Inactivation effects on CS cells in light}

Thirty-six CS cells were recorded from five animals during retrosplenial cortex inactivation in light testing conditions. Table 2 displays the absence of significant changes in reliability, information content, sparsity, and mean rate between control and inactivation trials of spatial memory performance. Spatial specificity showed a marginally significant decrease during inactivation $[t(35)=2.04 ; p=0.05]$. Spatial specificity, information content, and sparsity are all measures of place-field size, but only spatial specificity is sensitive to firing on single versus multiple arms across trials. Therefore, the significant decrease in spatial specificity may reflect place fields firing on multiple arms after inactivation of retrosplenial cortex (see further discussion of this issue below).

Twenty-four of the $36 \mathrm{CS}$ cells had place fields in either the control light or inactivation light trials (see description of placefield criteria in Materials and Methods of Experiment 1). For these cells, we examined changes in place coding during control and inactivation trials using the spatial correlation measure (see Fig. 2 and Behavioral and electrophysiological data analyses in Materials and Methods of Experiment 1). Despite the sustained behavioral accuracy during light inactivation, Figure $5 A$ displays the significant decrease in mean spatial correlation from con-tocon to con-to-inact testing conditions. The mean con-to-con correlation was $0.20( \pm 0.04)$, and it dropped to $0.10( \pm 0.02)$ in the con-to-inact correlation $[t(23)=2.61 ; p<0.02]$. To relate the change in spatial correlation of place cells to the behavior during inactivation, a Pearson correlation was computed between the con-to-inact spatial correlation and average errors per trial during the inactivation phase of testing. Figure $5 B$ displays the nonsignificant correlation between the spatial correlation scores and performance on the maze during light testing $[r(24)=0.28$; NS $]$.

To establish whether the place fields began to return to their original location during the inactivation condition, the spatial correlation that was computed between trials 1 and 2 of the control condition were compared with that of trials 9 and 10 of the inactivation condition. The spatial correlation was 0.15 ( \pm 0.04$)$, which was not significantly different from the control spatial correlation score $[t(23)=0.44 ; \mathrm{NS}]$. In the light trials, animals continued to run maze trials (trials 11-15); therefore we computed an additional spatial correlation between the first two control trials and the last two trials of the testing condition. The spatial correlation between these trials was 0.15 ( \pm 0.04$)$, which is comparable with the spatial correlation comparing the control light trials with the end of the inactivation trials. Thus, the recovery that occurs during the inactivation trials does not substantially change with additional maze trials. This suggests that the most pronounced changes in spatial coding after inactivation occur during the initial trials and that by the end of the inactivation condition the effects are less prevalent and do not change substantially with repeated maze trials. This pattern of data is consistent with previous work using injections of tetracaine and this testing procedure (Mizumori et al., 1989, 1994).

To examine further when the place fields are stable and unstable during inactivation of retrosplenial cortex, we computed the spatial correlation comparing individual trials with each other before and after the injection. For establishing the stability of control light trials, the spatial correlation between trials 1 and 2 of the control trials was calculated. To examine stability of spatial coding during and after inactivation of retrosplenial cortex, we computed the spatial correlation between the first two inactivation trials (trials 6 and 7) and the last two trials of the inactivation condition (trials 9 and 10). Between the first two baseline trials the spatial correlation was $0.29( \pm 0.06)$, during the first two inactivation trials the correlation was 0.13 ( \pm 0.04 ), and during the last two trials of the inactivation condition the correlation was 0.19 ( \pm 0.05$)$. A repeated measures ANOVA revealed that there was a significant change in the spatial correlation values observed across trials $\left[F_{(1,2)}=4.09 ; p<0.02\right]$. A Newman-Keuls post hoc analysis demonstrated that only the first two trials during inactivation were significantly different from the control trials or the last pair of inactivation trials $(p<0.05)$. These data suggest that inactivation of retrosplenial cortex caused initial instability of the location-coding properties of place fields, but they tend to regain spatially coherent firing after repeated maze trials.

The spatial correlation examined two inactivation trials compared with two control trials. However, the spatial firing measures presented in Table 2 were based on analyses between five maze trials. To ensure that the data presented in Table 2 have sufficient 
Table 1. $\theta$ Cell responses to inactivation during light and dark testing

\begin{tabular}{|c|c|c|c|c|}
\hline \multirow[b]{2}{*}{$\theta$ Cells $\left(n=11^{a}\right)$} & \multicolumn{2}{|l|}{ Light $(n=7)$} & \multicolumn{2}{|l|}{ Dark $(n=8)$} \\
\hline & Control & Inactivation & Control & Inactivation \\
\hline Spatial Spec. & $1.65 \pm 0.10$ & $1.77 \pm 0.18$ & $1.34 \pm 0.04$ & $2.68 \pm 0.93$ \\
\hline Rel & $0.31 \pm 0.07$ & $0.36 \pm 0.06$ & $0.41 \pm 0.07$ & $0.50 \pm 0.14$ \\
\hline Info Cont. & $0.67 \pm 0.13$ & $0.61 \pm 0.14$ & $0.68 \pm 0.18$ & $0.70 \pm 0.21$ \\
\hline Spars. & $0.53 \pm 0.06$ & $0.55 \pm 0.06$ & $0.54 \pm 0.06$ & $0.56 \pm 0.07$ \\
\hline Mean Rate & $13.70^{*} \pm 1.98$ & $15.02 \pm 2.17$ & $15.81 \pm 4.50$ & $16.46 \pm 4.69$ \\
\hline
\end{tabular}

The average spatial specificity, reliability, information content, sparsity, and mean rate for the $\theta$ cells recorded during light and dark inactivation are shown.

${ }^{a}$ The total number of cells does not match the sum of the numbers in the light and dark conditions because four $\theta$ cells were recorded in both conditions.

${ }^{*} p<0.05$.

Info Cont., Information content; Rel., reliability; Spars., sparsity; Spatial Spec., spatial specificity.

Table 2. CS cell responses to inactivation during light and dark testing

\begin{tabular}{|c|c|c|c|c|}
\hline \multirow[b]{2}{*}{ CS cells $\left(n=58^{a}\right)$} & \multicolumn{2}{|c|}{ Light $(n=36)$} & \multicolumn{2}{|c|}{$\underline{\text { Dark }(n=32)}$} \\
\hline & Control & Inactivation & Control & Inactivation \\
\hline Spatial Spec. & $7.32 \pm 1.12$ & $5.23 * \pm 0.86$ & $6.74 \pm 1.50$ & $6.53 \pm 1.92$ \\
\hline Rel & $0.47 \pm 0.04$ & $0.42 \pm 0.04$ & $0.36 \pm 0.04$ & $0.38 \pm 0.05$ \\
\hline Info Cont. & $3.85 \pm 0.40$ & $3.63 \pm 0.39$ & $4.61 \pm 0.35$ & $4.68 \pm 0.34$ \\
\hline Spars. & $0.05 \pm 0.02$ & $0.06 \pm 0.01$ & $0.06 \pm 0.01$ & $0.05 \pm 0.01$ \\
\hline Mean Rate & $0.71 \pm 0.14$ & $0.72 \pm 0.15$ & $0.65 \pm 0.15$ & $0.64 \pm 0.15$ \\
\hline
\end{tabular}

The average spatial specificity, reliability, information content, sparsity, and mean rate for the CS cells recorded during light and dark inactivation are shown.

${ }^{a}$ The total number of cells does not match the sum of the totals from the light and dark conditions because 10 CS cells were recorded in both conditions.

${ }^{*} p<0.05$.

resolution to detect changes in location coding during retrosplenial cortex inactivation, we performed the identical analysis comparing the first two control trials with the first two inactivation trials. There were no significant changes in spatial specificity, information content, sparsity, or mean rate (data not shown). It should be noted, however, that the two trial analyses might not provide sufficient resolution to detect changes in place fields.

The light inactivation effects could be caused by nonspecific factors, such as maze removal or stress from the injections. To confirm that this was not the case, 14 cells with place fields were tested with vehicle control injections. In these cases, the average correlation did not change as a function of injection. During the con-to-con phases of testing the spatial correlation was 0.18 ( \pm 0.02 ), and it was 0.22 ( \pm 0.04 ) during con-to-vehicle-control phases $[t(13)=-0.35$; NS]. This provides additional evidence that the change in the spatial correlation is likely caused by temporary inactivation of retrosplenial cortex and not caused by nonspecific effects of the injection procedure.

These data suggest that inactivation of retrosplenial cortex changes the preferred firing location of the place cells. Figure 6 displays two simultaneously recorded place cells during couplets of control trials, inactivation trials, and recovery trials (next day). The cell in Figure $6 A$ showed a preference for the west end of the maze arm during the control trials 1 and 2 and trials 4 and 5 . These trials were used for the con-to-con spatial correlation. During inactivation trials 6 and 7 , the original location of the place field changed to firing on the west and north arms of the maze. These trials were compared with the control trials 1 and 2 for the con-to-inact spatial correlation. The original location of the place field did not return during trials 9 and 10; however, the correlate did return on the following test day. In Figure $6 B$, a simultaneously recorded cell shows a response similar to that of the cell displayed in Figure $6 A$. The graphical display of the fields may appear somewhat "noisy" because the data displayed represent only two trials. During this time, the animal only passed through each maze arm a total of four times. This compares with displays in other reports that represent 20-30 min of sampling, and more visits, to particular locations (Mizumori et al., 1990, 1994).

\section{Inactivation effects on CS cells in dark}

Thirty-two CS cells from four animals were recorded during retrosplenial cortex inactivation in darkness. Eighteen CS cells were recorded during dark I inactivation, and fourteen CS cells were recorded in dark II inactivation. There were no significant differences in errors between the dark testing conditions, so the data were combined for the electrophysiological analyses. Similar to the light testing, spatial specificity, reliability, information content, sparsity, and mean rate did not change significantly between control and inactivation trials (Table 2). However, the preferred firing location of place cells did not remain stable during inactivation in darkness.

Of the $32 \mathrm{CS}$ cells tested during inactivation in darkness, 20 showed a place field in either the control or inactivation dark trials. For these 20 cells, we computed the spatial correlation between the con-to-con dark trials and the con-to-inact dark trials (Fig. $5 C$ ). The mean spatial correlation was $0.27( \pm 0.06)$ in the con-to-con condition and dropped significantly to $0.03( \pm 0.02)$ in the con-to-inact phase of testing $[t(19)=3.387 ; p<0.01]$. As with the light trials, we also computed a Pearson correlation between 

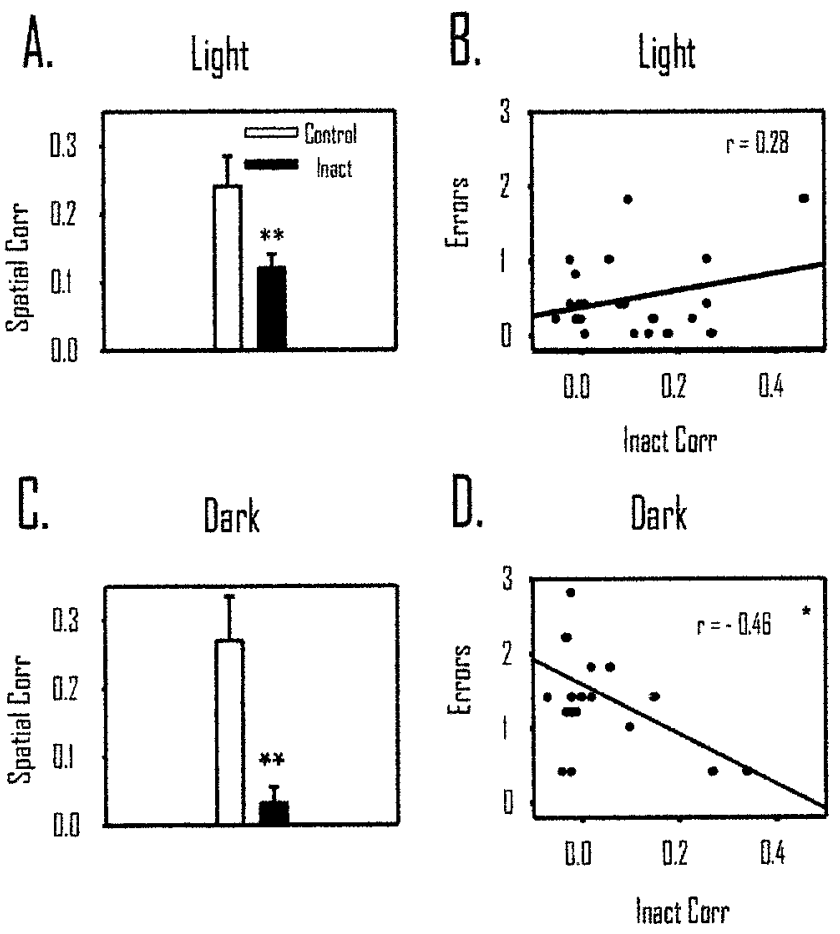

Figure 5. Temporary inactivation of retrosplenial cortex decreased after inactivation of retrosplenial cortex in light and dark but was only related to behavior during dark testing. $A$, The spatial correlation during con to con (Control) is significantly higher than that during con to inact (Inact; $p<0.01)$. This suggests that hippocampal place cells changed their preferred firing location during light inactivation. $B$, In light inactivation, there was no relationship between errors and the spatial correlation, suggesting that changes in place coding by hippocampal cells do not predict behavioral performance in the light. $C$, The spatial correlation in dark inactivation decreased significantly when retrosplenial cortex was inactivated $(p<0.01)$. $D$, There was a significant correlation between errors in darkness and changes in place coding by hippocampal place cells. ${ }^{*} p<0.05 ;{ }^{*} p<0.01$. Corr, Correlation.

the con-to-inact spatial correlation and errors committed during inactivation. Figure $5 D$ displays a negative relationship between errors and the spatial correlation $[r(20)=-0.46 ; p<0.05]$. The negative correlation suggests that animals made more errors when there was a larger change in hippocampal spatial coding (i.e., a lower spatial correlation). We analyzed the CS cells $(n=$ 5 ) recorded in the first dark testing session of each animal. These cells showed a pattern of data in the spatial correlation measure comparable with that shown by the remaining cells in subsequent dark trials.

As we did in the light trials, we compared the first two control trials with the first two inactivation trials for the measures of spatial firing by hippocampal place cells. Similar to the data collapsing across five trials, there were no significant changes between control and inactivation phases of testing for spatial specificity, information content, sparsity, or mean rate (data not shown). The absence of significant changes in place-field measures, but a significant change in the spatial correlation, suggests that the place fields changed their preferred firing location after inactivation of retrosplenial cortex.

As with the light trials, the duration of place-field changes during dark trials was evaluated by computing the spatial correlation between individual trials. The last two dark trials (dark I inactivation) or the first two dark trials (dark II inactivation) were used to establish control stability in darkness. The control stabil- ity was compared with the spatial correlation obtained from the first two trials after inactivation and with the spatial correlation from the last two trials of the dark inactivation condition. This trial-by-trial correlation provides information about the stability of the place fields between individual trials of the treatment conditions. The correlation for the baseline dark trials was 0.52 $( \pm 0.82$ ), during the first two trials after inactivation the correlation was $0.09( \pm 0.05)$, and during the last two trials of the dark inactivation condition the correlation was $0.33( \pm 0.09)$. A repeated measures ANOVA revealed that there was a significant change in the spatial correlation values across trials $\left[F_{(1,2)}=6.22\right.$; $p<0.05]$. A Newman-Keuls post hoc analysis showed that the spatial correlation during the first two inactivation trials was significantly different from that of both the control dark trials and the last trials of the inactivation condition $(p<0.05)$. These data suggest that, similar to light trials, there is a significant decrease in intertrial stability in spatial coding by place cells immediately after inactivation but that this instability is eventually replaced with a period of stability after several maze trials.

Two different cells that showed dark field reorganization are displayed in Figure 7, $A$ and $B$ (dark I and dark II injection procedures, respectively). In Figure $7 A$, the two light trials are shown for a cell with a field localized to the end of the north maze arm. The location of the preferred field was disrupted during the pairs of dark inactivation trials, trials 6 and 7 and trials 9 and 10 . The preferred location of the field did not return to the original location until the last pair of dark control trials (trials 14 and 15). Another cell recorded during the dark II inactivation procedure is displayed in Figure $7 B$. This place cell showed a preferred firing location on the northern edge of the center platform during light trials and was generally maintained during the pairs of dark trials used for the con-to-con spatial correlation analysis. In contrast to the center platform field location during control light and dark trials, after inactivation the field shifted to the west and southwest maze arms. The field slowly, although incompletely, began to return to the center platform during the final pair of dark inactivation trials. In all of the cases with dark testing on the subsequent day, the place field returned to the original control location.

\section{Comparison between light and dark}

A total of $10 \mathrm{CS}$ cells were tested in both light and darkness. Of these 10 cells, five CS cells showed place fields in both lighting conditions. To determine whether inactivation caused a greater change in place fields in darkness than in light, we compared the data from these five cells. The average control light spatial correlation was $0.22( \pm 0.11)$, and the control dark spatial correlation was 0.23 ( \pm 0.17$)$. A within-subjects $t$ test showed that these values were not significantly different $[t(4)=-0.05$; NS $]$. The average light inactivation spatial correlation was $0.10( \pm 0.05)$, and the average dark inactivation spatial correlation was -0.01 $( \pm 0.005)$. A within-subjects $t$ test showed that this difference between light and dark approached statistical significance $[t(4)=$ $2.65 ; p=0.06]$.

An example of a cell with a place field that showed a similar pattern of reorganization in light and dark, but greater reorganization in darkness, is displayed in Figure 8. This cell was recorded across several days and maintained a primary field in the light and dark on the southeast maze arm. Figure $8 A$ displays the effects of inactivation during light testing. The top spatial plot shows five control light trials, and the spatial plots below are the individual trials after inactivation of retrosplenial cortex (with the 
Figure 6. Responses of two simultaneously recorded place cells during light inactivation. Pairs of trials are shown to illustrate the spatially selective activity that occurred during the con-to-con and con-to-inact spatial correlation. $A$, In this case the cell showed a preferred field on the western maze arm during trials 1 and 2 and during trials 4 and 5 (con-to-con trials). During retrosplenial cortex inactivation, the location of the field changed to firing on two arms and then began to fire on the northwestern maze arm in the subsequent trials. The preferred location for the cell did not return until the subsequent test day. $B$, This cell showed a similar consistent field during control light trials; during inactivation the field changed locations and then began to fire on the southeastern maze arm. Interestingly, the simultaneously recorded cells both rotated their preferred fields during inactivation but by different amounts. In both cases, the preferred field did not return until the next test day. All of the spatial plots omit cellular activity that is $<20 \%$ of the maximum rate of the cell during the trials. The maximum rate is shown as dark areas, and shaded areas correspond to intermediate rates. This form of presentation is the same for Figures 7,8 , and 10 . It should be noted that the small sample size for the spatial plots reduces the variability in the firing of the place cell (the animal only passes through the place field a total of four times, and if the field is directional the cell only has two opportunities to be active for a given plot). Accordingly, the firing-rate distribution is reduced substantially because of the small sample size.
A. Contral Light
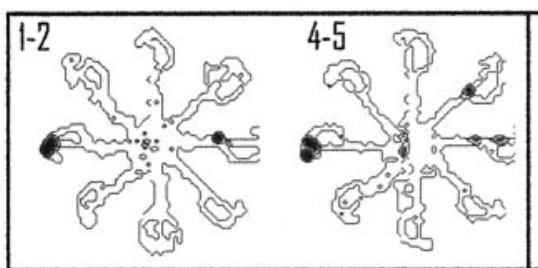

Inactivation Light

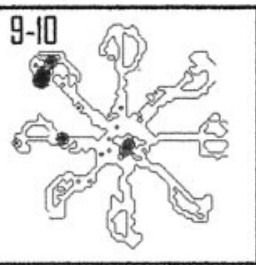

Next Day

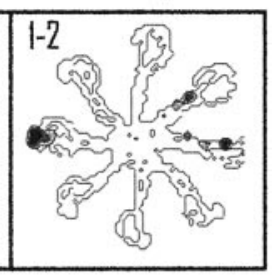

B. Contral Light Inactivation Light Next Day

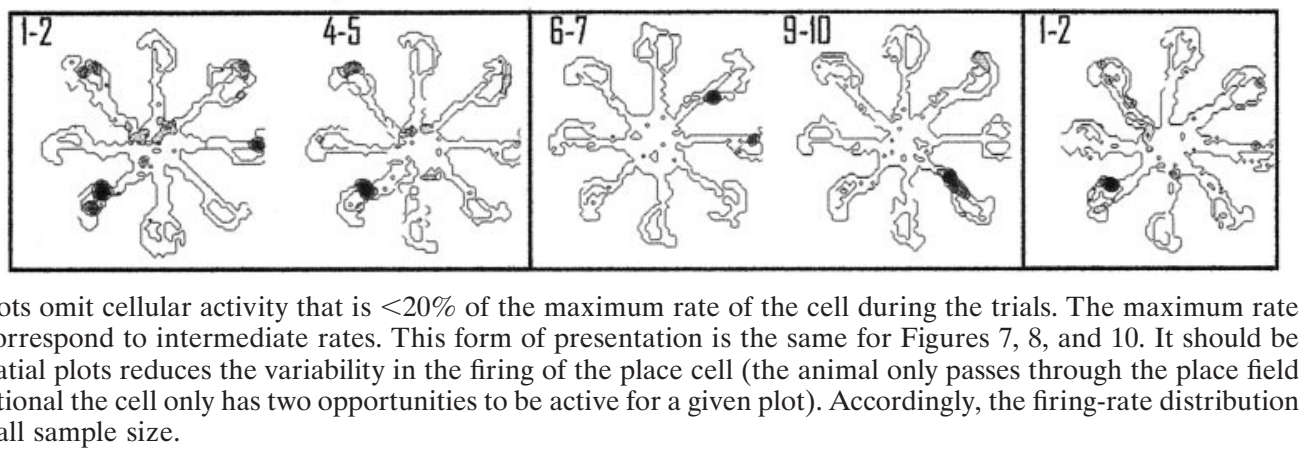

A. Light Dark Inactivation Dark Control

Figure 7. Responses of two different place cells recorded in dark I and dark II inactivation conditions. $A$, This cell showed a preferred field on the north maze arm during the initial light trials, and the preferred location changed across trials during the inactivation condition. The field did not return to the original location until the last pair of control dark trials. $B$, The preferred field of this cell was on the northern portion of the center platform during light trials and control dark trials. During inactivation, the field shifted to firing on two maze arms and began to return during the last inactivation trials.

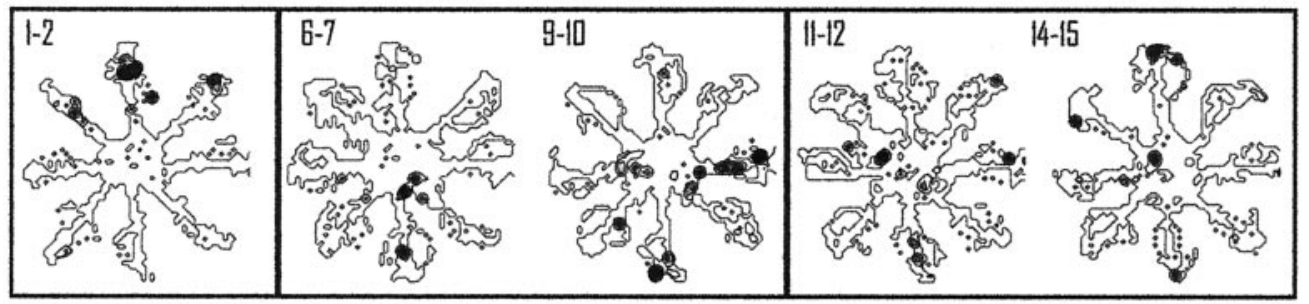

\section{B. Light}

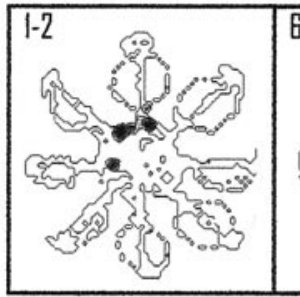

Dark Control

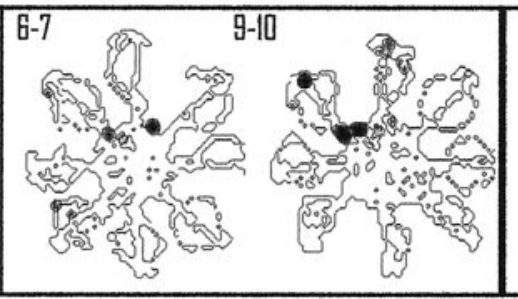

Dark Inactivation

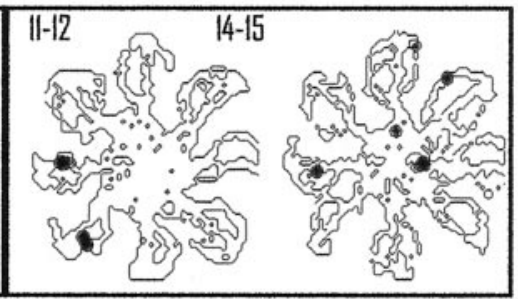

number next to the plot indicating the trial number after inactivation). During the first trial after light inactivation, the field shifted to the north arm of the maze. This location is maintained until the fourth light trial. During the fourth light trial, the field returned to the southeast arm of the maze and maintained that location for the subsequent trials (only one more trial is displayed in the figure).

In Figure $8 B$, the five light trials preceding the injection of tetracaine are displayed in the top spatial plot. As with the previous day, the field was located primarily on the southeast maze arm. During dark I inactivation, the field changed locations across the first two dark trials. On dark trial three, the place field began to stabilize on the north maze arm and maintained that preference for the majority of the trials in darkness (data not shown). This is consistent with the location of the field during light inactivation (shown in Fig. $8 A$ ). The original location of the field did not return to the southeast maze arm until the last trial in darkness (trial 10). For this cell, when visual information was available during retrosplenial cortex inactivation, the reorganization of spatial coding by hippocampal neurons was less persistent compared with testing without visual information.

\section{Stability across days}

The five cells that contributed to the light-dark comparison were examined for stability across days. Baseline spatial stability was assessed by calculating the spatial correlation for trials 1 and 2 


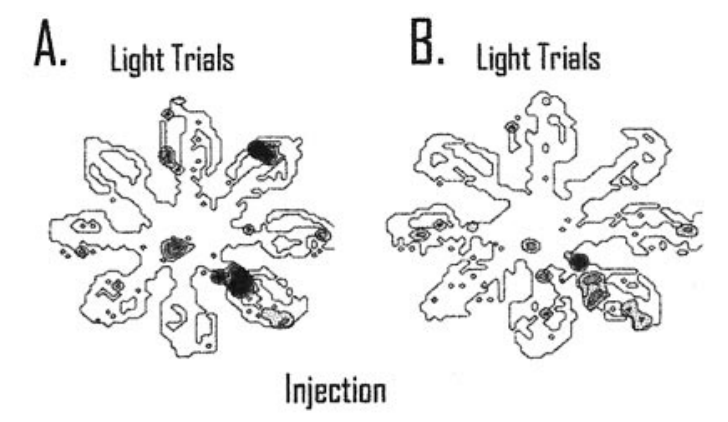

Light Trials
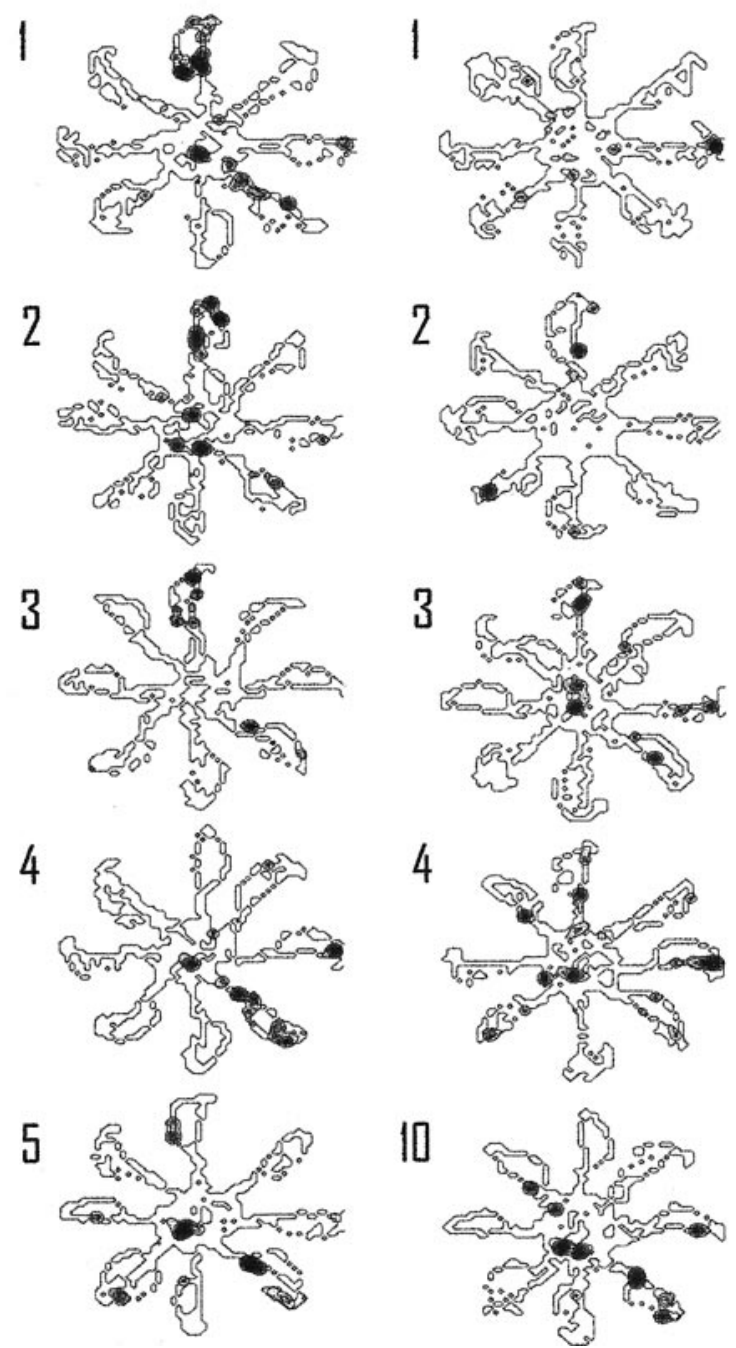

4
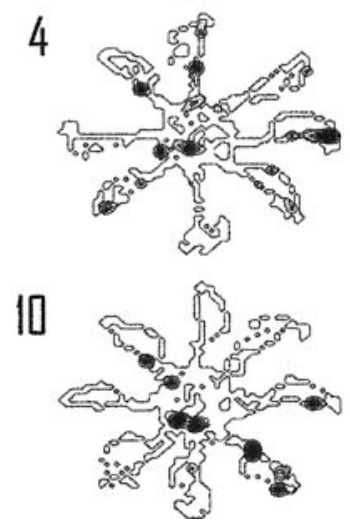

Figure 8. Recovery after inactivation requires more time in darkness than in light. The top spatial plots in $A$ and $B$ display five light trials preceding tetracaine injection. Individual trials after inactivation of retrosplenial cortex are displayed below the injection line; the number to the left of the spatial plot corresponds to the trial number after injection. $A$, After inactivation of retrosplenial cortex, the field shifted from the southeast maze arm to firing on the north arm of the maze and maintained that firing pattern until the fourth light trial. The field then maintained this location for the majority of the remaining light trials (only one more trial is displayed in the figure). $B$, For the dark I inactivation procedure, the cell did not show a consistent preferred firing location until the third dark trial after inactivation. For this trial and the majority of the remaining trials, the cell continued to fire on the north arm of the maze. This is the same location found to be the preferred firing pattern during light inactivation trials. The original preferred location for this cell did not return and trials 4 and 5 for day 1. To determine whether the spatial firing changed across days, the spatial correlation was computed between trials 1 and 2 of day 1 and trials 1 and 2 of day 2 . The baseline correlation for day 1 was $0.35( \pm 0.12)$, and the acrossdays correlation value was $0.36( \pm 0.20)[t(4)=-0.09$; NS $]$. Thus, the spatial-coding properties of place cells in hippocampal neurons are stable across days, suggesting that the changes in spatial coding after inactivation of retrosplenial cortex are transient.

\section{Partial versus complete reorganization}

It is presently unclear under what environmental conditions place fields develop entirely new location-selective codes (i.e., complete reorganization) or maintain similar, but not identical, spatial firing patterns (i.e., partial reorganization). To address this issue within the context of the contribution of retrosplenial cortex, we compared the spatial correlation measures in light and dark during inactivation for multiple simultaneously recorded place cells. We set an a priori criteria for "reorganization" as a spatial correlation score for a given cell that was less than the mean for all of the cells minus one SEM [e.g., reorganization = spatial correlation for a cell $<$ (mean of all cells - SEM)].

During light testing with inactivation of retrosplenial cortex, there were five data sets with multiple simultaneously recorded place cells (four to six cells per data set). In four of the five data sets, at least one cell did not show spatial reorganization, despite the fact that the remaining simultaneously recorded cells showed reorganization. Thus, not all cells responded in the same way during inactivation in light testing, which suggests that there was partial reorganization of spatial coding during light inactivation trials (for individual data during inactivation in light, see Fig. $5 B$ ). During dark inactivation, there were four data sets with multiple place cells recorded during inactivation of retrosplenial cortex (three to four cells per data set). In three of the four data sets, all of the cells recorded showed reorganization. However, in one data set, two of the four cells simultaneously recorded showed reorganization, and the remaining two did not. Interestingly, this one case occurred when inactivation of retrosplenial cortex did not result in behavioral impairments in darkness (see Fig. $5 D$ for individual cell responses during inactivation in dark). In sum, the data are consistent with the interpretation that there is partial, and not complete, reorganization during inactivation of retrosplenial cortex in light and dark testing.

\section{Experiment 2 Histology}

Six animals were used in this experiment. One animal assigned to the control injection group received a unilateral guide cannula placement. The data from this animal were virtually identical to those of the other control animals and were included in the control group. The injection sites for the remaining five animals were in the same areas displayed in Figure $3 A$ (four of the six animals were included in both experiments). Recording sites were in CA1 and CA3 of the hippocampus in regions similar to those displayed in Figure $3 B$. Approximately half of the cells in the tetracaine and control groups were in CA3 and CA1. Because of the small sample size, the data were combined for the analyses.

$\leftarrow$

until the last dark trial (10 trials later). Thus, without visual information to update the place-coding system, the cellular correlate requires more trials to return to the original location compared with when visual information is available. 
A.
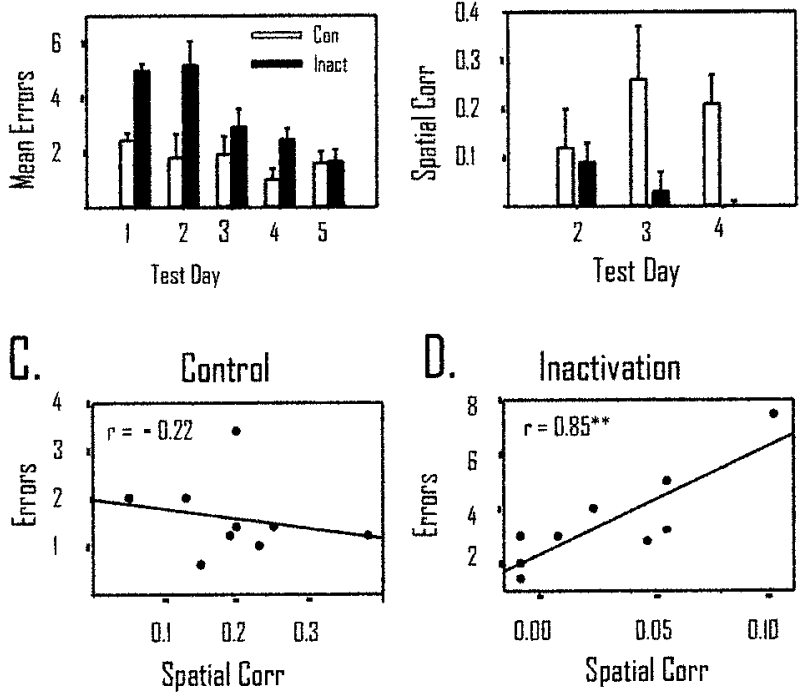

Figure 9. Inactivation of retrosplenial cortex impairs spatial learning and place-field stability. $A$, Spatial memory acquisition is impaired when tetracaine is infused into the retrosplenial cortex immediately before testing. The mean number of errors is significantly $(p<0.01)$ higher in the tetracaine group (Inact) compared with the control group (Con). There is a significant improvement across test days in both groups $(p<$ $0.05)$. $B$, The spatial correlation also shows a significant difference between tetracaine and control groups $(p<0.05)$. Only test days $2-4$ are displayed because those are the only days during which animals ran five trials and multiple hippocampal CS cells were recorded from the animals. Although there is a significant difference between groups in the spatial correlation, there was not a significant within-group effect of training. These data suggest that although behavior improves across trials, the spatial correlation does not. $C$, The spatial correlation did not relate to behavioral performance during acquisition in the animals receiving vehicle control injections $[r(9)=-0.22$; NS $]$. $D$, In contrast to control animals, tetracaine injections into retrosplenial cortex resulted in a highly significant correlation between errors and place-field stability $[r(9)=0.85 ; p<$ 0.01]. This suggests that when place fields remain in the same location across trials after inactivation of retrosplenial cortex there is a greater likelihood for behavioral impairments $(* * p<0.01)$.

\section{Behavioral data}

Two of the animals assigned to the control group ran two and three trials, respectively, during the first day of spatial memory training. During the next $4 \mathrm{~d}$, all of the control animals ran five spatial memory trials. All of the animals receiving tetracaine injections ran at least four trials on the first day of acquisition and five trials on the remaining $4 \mathrm{~d}$. The mean number of errors for each day is based on the number of trials that each animal performed during each test day. Figure $9 A$ displays the significant behavioral impairment during acquisition of the spatial memory task in light testing conditions. A mixed-design repeated measures ANOVA revealed a significant main effect of treatment group $\left[F_{(1,4)}=34.42 ; p<0.02\right]$, a significant within effect of training day $\left[F_{(1,4)}=5.30 ; p<0.02\right]$, but no significant interaction between group and training $\left[F_{(1,4)}=2.40 ; p>0.05\right]$. A NewmanKeuls post hoc analysis of the within effect showed that days 1 and 2 were significantly different from days 4 and $5(p<0.05)$. Therefore, tetracaine injections into retrosplenial cortex caused an initial performance deficit during spatial memory acquisition, but animals in both treatment groups were able eventually to acquire the task.

\section{Electrophysiological data}

In the inactivation group $(n=3)$, nine CS cells were recorded on day 2 of acquisition, seven were recorded on day 3 , and six were recorded on day 4 . In the control group $(n=3), 13$ cells were recorded on day 2 of acquisition, 12 were recorded on day 3 , and 10 were recorded on day 4 . In agreement with the data obtained from experiment 1 , inactivation of retrosplenial cortex did not result in significant changes between groups in spatial specificity, reliability, information content, sparsity, or mean rate during the $3 \mathrm{~d}$ of acquisition (see Table 3 ). In contrast to the absence of changes in these measures, Figure $9 B$ displays the significant decrease across $3 \mathrm{~d}$ of acquisition for the spatial correlation of CS cells in the inactivation group compared with the control group. A mixed-design ANOVA revealed a significant main effect of treatment group $\left[F_{(1,4)}=21.37 ; p=0.01\right]$ and interaction of group by training $\left[F_{(1,4)}=5.14 ; p<0.05\right]$ but not a significant within effect of training $\left[F_{(1,4)}=0.84 ; \mathrm{NS}\right]$.

To relate the spatial correlation scores with behavioral performance for each animal, we computed a Pearson correlation between the average spatial correlation score for each day and the average number of errors committed on each test day. Thus, there were nine correlation scores for the control and inactivation groups. A negative correlation between errors and the spatial correlation score would be indicative of improved performance with increasing stability of hippocampal representations of space. Figure $9 C$ displays the absence of a significant relationship between errors and the spatial correlation $[r(9)=-0.22$; NS]. For the inactivation group, there was a significant correlation between the spatial correlation and errors $[r(9)=0.85 ; p<0.01]$ (Fig. 9D). The positive correlation suggests that field stability in the inactivation group is indicative of poorer performance.

In the tetracaine group, only seven CS cells were recorded across $2 \mathrm{~d}$, and of those three were recorded for 3 consecutive days. Five CS cells in the control group were recorded for 3 continuous days. An interesting pattern was observed in cells recorded from animals in the inactivation group; all of the cells showed different preferred firing locations across days (as measured by the maximal rate on one arm of the maze across the five trials). In contrast, only two of the place fields recorded from control animals changed their preferred spatial firing across days. Figure $10 A$ displays a cell from the inactivation group that showed a different preferred location across days. Figure $10 \mathrm{~B}$ displays a cell from the control group across days $2-4$ of acquisition that remained in the same location across days.

\section{DISCUSSION}

\section{Experiment 1}

The present results confirmed our previous findings that inactivation of retrosplenial cortex impairs spatial memory performance in darkness. Retrosplenial cortex inactivation also caused hippocampal place cells to change their spatial firing patterns in light and dark testing conditions. The change in spatial coding occurred in the presence, and absence, of behavioral impairments (this issue will be explored further in General Discussion).

Nonspecific effects of the injection are not likely explanations for the behavioral and electrophysiological changes. First, vehicle control injections did not change hippocampal spatial coding in the light. In addition, instability in single-cell recording is not a potential cause of the observed changes in spatial coding, because the original place field of the cell returned during control trials or the subsequent day. Spread of tetracaine into the hippocampus is 


\begin{tabular}{|c|c|c|c|c|}
\hline Measure & & Day 2 & Day 3 & Day 4 \\
\hline \multirow[t]{2}{*}{ Spatial Spec. } & Tet & $2.55( \pm 0.27)$ & $3.27( \pm 1.11)$ & $2.51( \pm 0.42)$ \\
\hline & Con & $3.89( \pm 1.03)$ & $3.90( \pm 1.03)$ & $5.45( \pm 2.44)$ \\
\hline \multirow[t]{2}{*}{ Rel } & Tet & $0.40( \pm 0.13)$ & $0.45( \pm 0.09)$ & $0.44( \pm 0.28)$ \\
\hline & Con & $0.46( \pm 0.127)$ & $0.50( \pm 0.09)$ & $0.41( \pm 0.12)$ \\
\hline \multirow[t]{2}{*}{ Info Cont. } & Tet & $2.20( \pm 1.27)$ & $2.60( \pm 0.43)$ & $2.37( \pm 0.70)$ \\
\hline & Con & $3.86( \pm 0.48)$ & $3.09( \pm 0.52)$ & $3.93( \pm 0.40)$ \\
\hline \multirow[t]{2}{*}{ Spars. } & Tet & $0.20( \pm 0.08)$ & $0.15( \pm 0.03)$ & $0.16( \pm 0.06)$ \\
\hline & Con & $0.13( \pm 0.05)$ & $0.19( \pm 0.07)$ & $0.08( \pm 0.005)$ \\
\hline \multirow[t]{2}{*}{ Mean Rate } & Tet & $3.35( \pm 2.34)$ & $1.56( \pm 0.54)$ & $1.09( \pm 0.31)$ \\
\hline & Con & $1.62( \pm 0.59)$ & $4.22( \pm 1.91)$ & $2.76( \pm 1.49)$ \\
\hline
\end{tabular}

The measures of spatially selective firing and mean firing rate during days 2-4 of spatial memory acquisition for the tetracaine and vehicle control groups are shown.

Con, Vehicle control group; Tet, tetracaine group.
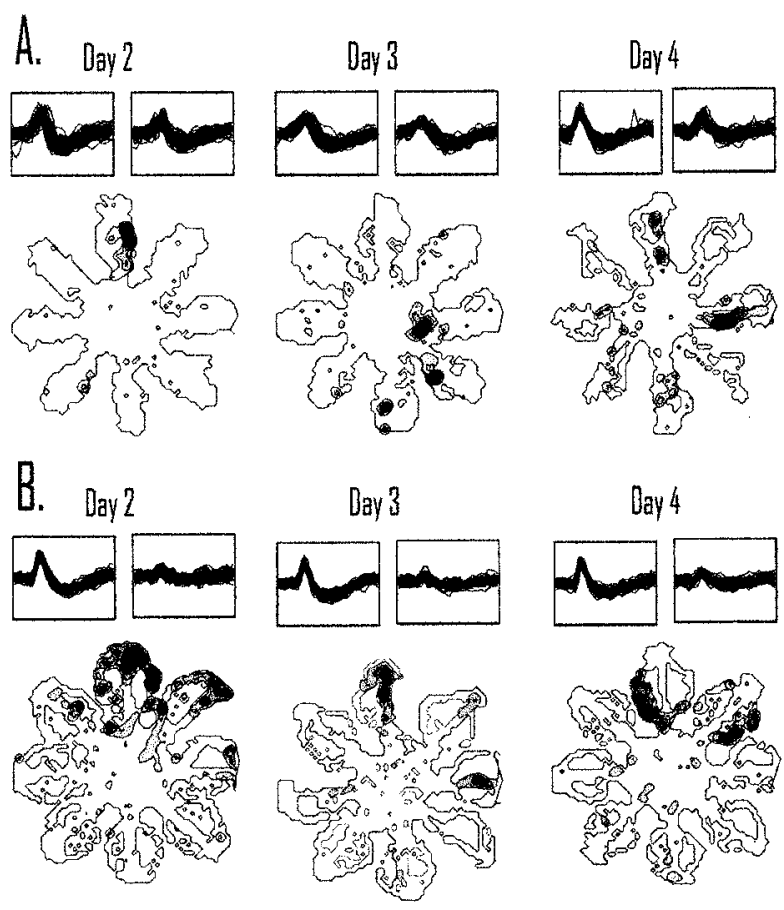

Figure 10. Inactivation of retrosplenial cortex causes place fields to be less stable across days. $A$, The location of a place field recorded across 3 consecutive days of acquisition recorded from an animal undergoing retrosplenial cortex inactivation is shown. The field changes the preferred firing location across days from the north arm to the eastern edge of the center platform and to the east maze arm on days $2-4$ of acquisition. $B$, Most of the cells recorded from an animal receiving vehicle control injections remained in the same location across days. The place field remains on the northern arm for 3 consecutive days of acquisition. Place fields appeared less stable after tetracaine injections than what was observed for animals receiving control injections. To illustrate recording stability, a set of 50 waveforms from each day is displayed above each spatial plot.

not a likely cause of the observed effects because the mean rate of the cells did not change significantly and dye was not observed in the hippocampus after ink injections. Motor disturbances are an unlikely explanation for the current results because maze run time did not change as a function of injections and retrosplenial cortex inactivation had no effect on performance during light trials.
A disruption in processing local maze cues is not a probable source of the dark performance deficit. Permanent removal of retrosplenial cortex results in behavioral impairments on the water maze, a task that substantially reduces the prevalence of local maze cues (Sutherland et al., 1988; Sutherland and Hoesing, 1993). In addition, head-direction cells are observed in retrosplenial cortex, and they are controlled by visual and movementrelated information rather than local cues (Chen et al., 1994b).

The cingulum bundle and Oc2MM were probably affected by the injections. However, inactivation of the adjacent cingulum bundle may not be responsible for the behavioral and electrophysiological changes, because permanent removal of the cingulum bundle, but not the retrosplenial cortex, impairs spatial memory performance on the radial maze in light conditions (Neave et al., 1997). Posterior parietal cortex integrates information from the external environment with motor movements (Colby and Goldberg, 1999). It has been suggested that movement-based information from posterior parietal cortex is transformed to an extended spatial coordinate reference frame in retrosplenial cortex (Vogt and Miller, 1983; Chen et al., 1994b). Retrosplenial cortex receives information from Oc2MM and has anatomical connections with the hippocampus via the entorhinal cortex and postsubiculum (Vogt and Miller, 1983; Wyss and van Groen, 1992). Accordingly, we suggest that the most parsimonious explanation of the current data is that retrosplenial cortex provides experiencedependent spatial information relevant for spatial memory performance in darkness and hippocampal spatial processing.

Behavioral studies suggest that dark performance on the radial maze may require the integration of mnemonic information, self-motion cues, and static background cues to solve the task (Brown and Bing, 1997). Save (1997) has demonstrated that water maze performance in darkness is enhanced with longer preexposure to the visual cues. This suggests that memory of the visual environment improves spatial localization in darkness. We suggest that when there is a strong requirement for spatial memory to guide processing of self-motion information (as is the case during dark performance), retrosplenial cortex plays a particularly important role in navigational accuracy. When visual cues are available, animals may rely on currently viewed spatial features of the environment to navigate accurately.

Navigation relying on nonvisual information is thought to be dependent primarily on processing movement-related cues. This type of navigation is referred to as path integration and is subject 
to cumulative error without an external source of information to update the navigational system (Gallistel, 1990; Etienne, 1992). One manipulation for disrupting self-motion cues (a component of the path integration process) is to carry an animal in an opaque box and slowly rotate the box while transporting the rat to the testing room. This manipulation causes spatial-learning impairments and place fields to be unstable across testing sessions (Knierim et al., 1995; Dudchenko et al., 1997; Martin et al., 1997). These data suggest that self-motion cues may be the metric for establishing stable representations of the visual environment (cf. McNaughton et al., 1996; Samsonovich and McNaughton, 1997; but see Redish and Touretzky, 1997). These results illustrate the importance of the normal integration of visual and self-motion cues for ensuring stable place coding and normal spatial learning.

During initial learning, animals may navigate by comparing their current position, direction, and velocity relative to remembered locations in space. This may be similar to the proposed role of memory in updating movement information when testing occurs in darkness (Brown and Bing, 1997; Save, 1997). If retrosplenial cortex contributes to the integration of mnemonic spatial information with self-motion cues, then inactivation of retrosplenial cortex would be expected to disrupt initial learning about visual environments. In addition, place fields would be expected to be less stable during acquisition in animals undergoing inactivation of retrosplenial cortex. We sought to explore our hypothesis further by examining behavioral and electrophysiological changes after inactivation of retrosplenial cortex during acquisition of the spatial memory task with room lights available.

\section{Experiment 2}

Experiment 2 revealed that retrosplenial cortex is necessary for normal performance of the spatial memory task with visual cues available. Furthermore, place-field stability within and across days of testing is dependent, in part, on the normal activity of retrosplenial cortex. If place fields in tetracaine-treated animals remained in the same location across trials within each day of acquisition, then performance was impaired (Fig. 9D). This pattern of data paralleled the behavioral data that demonstrated that animals in the tetracaine group were eventually able to acquire the spatial memory task. Unreliable spatial coding within a day is a unique phenomenon because it is commonly assumed that stable coding of space leads to more accurate performance (Mizumori et al., 1994, 1996; Barnes et al., 1997). However, the current testing conditions are not directly analogous to previous work. First, animals are performing a task while a brain area is temporarily inactivated, and second, the effectiveness of tetracaine likely decreases with time (see Experiment 1). The small sample size limits broad conclusions, but the finding that performance on the maze improved across days suggests that there may be behavioral and neural compensation. This compensation, in turn, may be reflected by this unique pattern of hippocampal spatial coding. If animals use the same location code established in the first trials after the inactivation condition during all of the maze trials, then they are less likely to navigate accurately. However, if there is a different spatial firing pattern in the later trials compared with that of the first two trials, animals are able to perform as well as controls. These data provide interesting evidence of future research evaluating how hippocampal processing of space is modified by behavioral or neural compensation during learning. Furthermore, they provide evidence of flexible hippocampal processing enabling spatial learning.

Previous lesion studies have demonstrated that permanent re- moval of retrosplenial cortex impairs spatial learning and memory in a water maze (Sutherland et al., 1988; Sutherland and Hoesing, 1993; but see Warburton et al., 1998) and disrupts orientation responses (Ellard et al., 1990; Kwon et al., 1990; Ellard and Chapman, 1991). However, retrosplenial cortex lesions do not result in spatial memory deficits on the eight-arm radial maze task when room lights are available (Neave et al., 1997). This provides an apparent paradox in the contribution of retrosplenial cortex to spatial learning and memory. Perhaps the paradox can be resolved by comparing differences in the requirement for visual memory and self-motion integration across tasks. The water maze is relatively devoid of local cues for use during spatial localization. Therefore, identifying locations based on movement relative to previously experienced visual cues is critical for solving this task. Orientation responses are typically elicited with visual stimuli and examine movement toward (approach) or away from (avoidance) the stimulus. Avoidance responses, in particular, may use spatial memory for recalling locations of potential refuge within the environment, and this mnemonic information may be integrated with the visual threat stimulus to mediate rapid flight responses. The radial maze in darkness is solved by a combination of mnemonic spatial cues, self-motion information, and local cues (Brown and Bing, 1997). The pattern of deficits across the tasks suggests that the integration of visual memory and movement-related cues is a common element underlying impairments after temporary or permanent damage to retrosplenial cortex.

Taken together, experiments 1 and 2 suggest that the retrosplenial cortex and hippocampus are interactive partners mediating spatial memory and behavior. Retrosplenial cortex importantly contributes to behavior when recalled information about the spatial environment needs to be integrated with self-motion cues to learn a spatial task. The reorganization of hippocampal spatial coding observed in the present experiments is consistent with previous work showing changes in spatial coding after manipulations of visual and self-motion cues (Sharp et al., 1995; Knierim et al., 1998) and with computational models that place these cues in conflict with each other (Guazzelli et al., 1999; Redish and Touretzky, 1999). These issues are developed below.

\section{General discussion}

Experiment 1 confirmed that retrosplenial cortex inactivation selectively impairs dark spatial memory performance, and experiment 2 showed that retrosplenial cortex is necessary for initial visual spatial learning. In contrast to the selective pattern of behavioral effects, hippocampal spatial coding was altered when performance was normal (Experiment 1, light testing) and impaired (Experiment 1, dark testing; Experiment 2, acquisition in the light). Hippocampal reorganization, as reflected by individual neurons, does not necessarily predict behavioral impairments. Instead, changes in hippocampal spatial coding may be related to modifications in the reliance on sensory modalities relevant for different cognitive strategies used to solve the task (cf. Markus et al., 1995). We suggest that the pattern of data from both experiments is consistent with the hypothesis that retrosplenial cortex contributes to the integration of visual and self-motion cues for use in navigation and for hippocampal spatial coding. When place cells change their reliance on sensory information for spatial coding, hippocampal reorganization likely occurs. Without the normal integration of visual and movement-related cues, animals may use different sources of sensory information for navigation and hippocampal spatial coding. 
It is possible that the hippocampal reorganization observed in the present experiments was caused by the entire visual environment being perceived as novel after inactivation of retrosplenial cortex. The novelty explanation appears unlikely. Animals transferred to a novel environment tend to make more errors (in the light) than they do in a familiar environment at asymptote levels of performance (Mizumori et al., 1995), which was not observed in the current study. Nonspecific sensory and motor deficits are not probable explanations for the observed effects. Changes in spatial coding did not occur during vehicle control injections, performance was preserved during light testing at asymptote, and maze run time did not change as a function of injections.

\section{Visual and self-motion integration, place-field reorganization, and behavior}

Knierim et al. (1998) placed visual and self-motion cues in conflict with each other by rotating the animal relative to a previously stable visual cue and recorded place cells before and after the rotation. When the rotation was $<45^{\circ}$, place cells followed the new location visual cue; when the rotation was $>45^{\circ}$, many place cells unpredictably changed their normal spatial firing patterns relative to the distal cue. Thus, when the perceived landmarks are mildly incongruent with the expected orientation of the animal, hippocampal place cells and perhaps the navigational system in general correct and update in agreement with the cues. If the landmarks are substantially deviated from the remembered configuration, then hippocampal cells show spatial reorganization. Similar results have been demonstrated in behavioral studies of the golden hamster. Rotation of a single visual landmark can control homing behavior when it is rotated $<45^{\circ}$ from the standard location. However, rotations by $>45^{\circ}$ caused animals to rely on internal sources of information to compute the return trajectory (Etienne et al., 1990). These experiments taken together with the current results suggest that (1) the hippocampus may reorganize spatial representations when there is substantial deviation between visual and self-motion cues and (2) despite the presence of hippocampal reorganization, behavior may be intact because animals can rely on either visual or self-motion cues to mediate navigation.

In addition to changes in place fields during light testing, there was an even more pronounced reorganization when retrosplenial cortex was inactivated before dark trials. This suggests that with visual cues available, the navigational system may correct and update more quickly than when they were not visible (see Fig. 8). It remains a possibility that inactivation of retrosplenial cortex may cause a deficit based solely on self-motion processing. This appears unlikely because vestibular and visual information are integrated in brainstem levels of the CNS (for review, see Smith, 1997), and the integration is preserved in all levels of the limbic system (for review, see Taube, 1998). Therefore, it appears more likely that retrosplenial cortex contributes to the integrative process rather than a single domain in isolation.

\section{Spatial memory and place-field reorganization}

Recent theories of navigation have suggested that during initial learning animals establish visuospatial representations relative to their movement through space (McNaughton et al., 1996; Samsonovich and McNaughton, 1997). Accordingly, we examined the role of retrosplenial cortex during visuospatial learning. Performance deficits were observed during initial learning after retrosplenial cortex inactivation (Experiment 2). In addition, place fields showed reorganization across trials and across days during acquisition. Previous work has demonstrated that normal integration of visual and self-motion cues is critical for appetitively mediated spatial learning and for establishing the preferred firing fields of hippocampal place cells (Knierim et al., 1995; Dudchenko et al., 1997; Martin et al., 1997). The results from experiment 2 are consistent with the interpretation that retrosplenial cortex provides spatial memory for use in guiding and directing movements during spatial learning and hippocampal coding of space.

In agreement with our interpretation that memory deficits may account for the hippocampal reorganization observed in the current experiments, Barnes et al. (1997) suggested that impaired spatial memory in aged animals might be related to changes in hippocampal reorganization. Hippocampal place coding in old animals periodically reorganized when they were reintroduced to a familiar environment (Barnes et al., 1997). The bimodal distribution of place-field responses may explain the behavioral performance of old animals on the water maze task, in which they displayed either accurate or inaccurate paths to the hidden platform (Barnes et al., 1997). Redish and Touretzky (1999) modeled this phenomenon by weakening the long-term potentiationdependent integration of visual cues with the resetting of path integration when simulated animals were introduced to the environment. In their computational model, a similar pattern of periodic spatial recoding of a familiar environment was observed. The experiment and the model are both consistent with our interpretation that spatial memory impairments may disrupt the normal integration of visual and self-motion cues and lead to hippocampal reorganization.

\section{Unique contribution of retrosplenial cortex to navigation}

Anatomical findings and experimental results suggest that there is a thalamic differentiation of visual and self-motion processing that can be relayed to retrosplenial cortex (van Groen and Wyss, 1992, 1995; van Groen et al., 1993). The lateral dorsal nucleus of the thalamus (LDN) and ATN may be related to visual and vestibular processing, respectively, of the head-direction signal. The LDN head-direction cells are visually sensitive, because they do not maintain their preferred firing direction for extended periods of time in darkness (Mizumori and Williams, 1993). In contrast, ATN head-direction cells are dependent on vestibular input (Blair and Sharp, 1996; Stackman and Taube, 1997) and persist for a substantial amount of time in darkness (Knierim et al., 1998). On-line updating of visual directional information may be processed through LDN input to retrosplenial cortex, whereas changes in directional heading derived from vestibular activation are relayed to retrosplenial cortex from ATN. Posterior parietal cortex could provide proprioceptive feedback to retrosplenial cortex.

Memory-guided navigation may be accomplished via a comparison between the currently experienced visual, vestibular, and proprioceptive inputs relative to past experiences within the environment. This comparison may allow for updating of the directional heading relative to the currently experienced sensory information (Mizumori et al., 2001). The mnemonic spatial information from retrosplenial cortex may then be relayed to the hippocampus directly via entorhinal input or indirectly through the postsubiculum (van Groen and Wyss, 1990b; Wyss and van Groen, 1992). Retrosplenial-hippocampal interactions may be critical for behavior when spatial localization requires mnemonic information about the environment.

It has been suggested that the hippocampus functions as a "path integrator" and allows animals to navigate successfully on the basis of movement through space (Maaswinkel et al., 1999; 
Whishaw and Gorny, 1999; but see Alyan and McNaughton, 1999) and that path integration information may be received from posterior cortical areas (Chen et al., 1994b; McNaughton et al., 1996; Guazzelli et al., 1999). In this study, changes in hippocampal coding of space were correlated with behavioral performance when path integration strategies may have been used (e.g., darkness and acquisition). However, there are examples when the recoding of space occurred despite high levels of performance (e.g., light testing). Therefore, the present results neither confirm nor reject the possibility that the hippocampus contributes to path integration. We suggest that although the hippocampus may be involved in navigation based on nonvisual cues, it likely does so in concert with retrosplenial cortex.

\section{Summary and conclusions}

The goals of the present study were to replicate and extend our previous findings of dark-selective behavioral impairments after inactivation of retrosplenial cortex and to examine putative neural mechanisms of the behavioral impairments. Retrosplenial cortex may contribute to navigation when animals are required to update spatial information relative to remembered features of the environment. This process likely requires interactions between the retrosplenial cortex and hippocampus as evidenced by changes in spatial coding when retrosplenial cortex was not active. Furthermore, the duration of hippocampal reorganization appears to be more persistent when visual cues are not immediately available to update the navigational system. Therefore, retrosplenial cortex interactions with the hippocampus may allow past experience to update and correct cumulative errors that occur during path integration.

\section{REFERENCES}

Alyan S, McNaughton BL (1999) Hippocampectomized rats are capable of homing by path integration. Behav Neurosci 113:19-31.

Barnes CA, Suster MS, Shen J, McNaughton BL (1997) Multistability of cognitive maps in the hippocampus of old rats. Nature 388:272-275.

Blair HT, Sharp PE (1996) Visual and vestibular influences on headdirection cells in the anterior thalamus of the rat. Behav Neurosci 110:643-660

Brown MF, Bing MN (1997) In the dark: spatial choice when access to spatial cues is restricted. Anim Learn Behav 25:21-30.

Chen LL, Lin LH, Green EJ, Barnes CA, McNaughton BL (1994a) Head-direction cells in the rat posterior cortex. I. Anatomical distribution and behavioral modulation. Exp Brain Res 101:8-23.

Chen LL, Lin LH, Barnes CA, McNaughton BL (1994b) Head-direction cells in the rat posterior cortex. II. Contributions of visual and ideothetic information to the directional firing. Exp Brain Res 101:24-34.

Colby CL, Goldberg ME (1999) Space and attention in parietal cortex. Annu Rev Neurosci 22:319-349.

Cooper BG, Mizumori SJY (1999) Retrosplenial cortex inactivation selectively impairs navigation in darkness. NeuroReport 10:625-630.

Cooper BG, Miya DY, Mizumori SJY (1998) Superior colliculus and active navigation: role of visual and non-visual cues in controlling cellular representations of space. Hippocampus 8:340-372.

Dudchenko PA, Goodridge JP, Seiterle DA, Taube JS (1997) Effects of repeated disorientation on the acquisition of spatial tasks in rats: dissociation between the appetitive radial arm maze and aversive water maze. J Exp Psychol Anim Behav Process 23:194-210.

Ellard CG, Chapman DG (1991) The effects of posterior cortical lesions on responses to visual threats in the Mongolian gerbil (Meriones unguiculatus). Behav Brain Res 44:163-167.

Ellard CG, Stewart DJ, Donaghy S, Vanderwolf CH (1990) Behavioural effects of neocortical and cingulate lesions in the Mongolian gerbil. Behav Brain Res 36:41-51.

Etienne AS (1992) Navigation of small mammal by dead reckoning and local cues. Am Psychol Soc 1:48-52.

Etienne AS, Teroni E, Hurni C, Portenier V (1990) The effect of a single light cue on homing behavior of the golden hamster. Anim Behav 39:17-41.

Fox SE, Ranck Jr JB (1975) Localization and anatomical identification of theta and complex spike cells in dorsal hippocampal formation of rats. Exp Neurol 49:299-313.
Fox SE, Ranck Jr JB (1981) Electrophysiological characteristics of hippocampal complex-spike cells and theta cells. Exp Brain Res 41:399-410.

Gallistel CR (1990) The organization of learning. Cambridge, MA: MIT.

Gothard KM, Skaggs WE, McNaughton BL (1996) Dynamics of mismatch correction in the hippocampal ensemble code for space: interaction between path integration and environmental cues. J Neurosci 16:8027-8040.

Guazzelli A, Bota M, Arbib MA (1999) Incorporating path integration capabilities in the TAM-WG model of rodent navigation. Neurocomputing 26:713-719.

Hill AJ, Best PJ (1981) Effects of deafness and blindness on the spatial correlates of hippocampal unit activity in the rat. Exp Neurol 74:204-217.

Jeffery KJ, O'Keefe JM (1999) Learned interaction of visual and idiothetic cues in the control of place field orientation. Exp Brain Res 127:151-161.

Jeffery KJ, Donnett JG, Burgess N, O'Keefe JM (1997) Directional control of hippocampal place fields. Exp Brain Res 117:131-142.

Knierim JJ, Kudrimoti HS, McNaughton BL (1995) Place cells, head direction cells, and the learning of landmark stability. J Neurosci 15:1648-1659.

Knierim JJ, Kudrimoti HS, McNaughton BL (1998) Interactions between idiothetic cues and external landmarks in the control of place cells and head direction cells. J Neurophysiol 80:425-446.

Kwon SE, Nadeau SE, Heilman KM (1990) Retrosplenial cortex: possible role in habituation of the orienting response. J Neurosci 10:3559-3563.

Maaswinkel H, Jarrard LE, Whishaw IQ (1999) Hippocampectomized rats are impaired in homing by path integration. Hippocampus 9:553-561.

Markus EJ, Barnes CA, McNaughton BL, Gladden VL, Skaggs WE (1994) Spatial information content and reliability of hippocampal CA1 neurons: effects of visual input. Hippocampus 4:410-421.

Markus EJ, Qin YL, Leonard B, Skaggs WE, McNaughton BL, Barnes CA (1995) Interactions between location and task affect the spatial and directional firing of hippocampal neurons. J Neurosci 15:7079-7094.

Martin GM, Harley CW, Smith AR, Hoyles ES, Hynes CA (1997) Spatial disorientation blocks reliable goal location on a plus maze but does not prevent goal location in the morris maze. J Exp Psych Anim Behav Process 23:183-193.

McNaughton BL, Barnes CA, O'Keefe J (1983a) The contributions of position, direction, and velocity to single unit activity in the hippocampus of freely-moving rats. Exp Brain Res 52:41-49.

McNaughton BL, O'Keefe J, Barnes CA (1983b) The stereotrode: a new technique for simultaneous isolation of several single units in the central nervous system from multiple unit records. J Neurosci Methods 8:391-397.

McNaughton BL, Barnes CA, Gerrard JL, Gothard K, Jung MW, Knierim JJ, Kudrimoti H, Qin Y, Skaggs WE, Suster M, Weaver KL (1996) Deciphering the hippocampal polyglot: the hippocampus as a path integration system. J Exp Biol 199:173-185.

Mizumori SJY, Williams JD (1993) Directionally selective mnemonic properties of neurons in the lateral dorsal nucleus of the thalamus of rats. J Neurosci 13:4015-4028.

Mizumori SJY, McNaughton BL, Barnes CA, Fox KB (1989) Preserved spatial coding in hippocampal CA1 pyramidal cells during reversible suppression of CA3c output: evidence for pattern completion in hippocampus. J Neurosci 9:3915-3928.

Mizumori SJY, Perez GM, Alvarado MC, Barnes CA, McNaughton BL (1990) Reversible inactivation of the medial septum differentially affects two forms of learning in rats. Brain Res 528:12-20.

Mizumori SJY, Barnes CA, McNaughton BL (1992) Differential effects of age on subpopulations of hippocampal theta cells. Neurobiol Aging 13:673-679.

Mizumori SJY, Miya DY, Ward KE (1994) Reversible inactivation of the lateral dorsal thalamus disrupts hippocampal place representation and impairs spatial learning. Brain Res 644:168-174.

Mizumori SJY, Garcia PA, Raja MA, Volpe BT (1995) Spatial- and locomotion-related neural representation in rat hippocampus following long-term survival from ischemia. Behav Neurosci 109:1081-1094.

Mizumori SJY, Lavoie AM, Kalyani A (1996) Redistribution of spatial representation in the hippocampus of aged rats performing a spatial memory task. Behav Neurosci 110:1006-1016.

Mizumori SJY, Ragozzino KE, Cooper BG, Leutgeb S (1999) Hippocampal representational organization and spatial context. Hippocampus 9:444-451.

Mizumori SJY, Cooper BG, Leutgeb S, Pratt WE (2001) A neural systems analysis of adaptive navigation. Mol Neurobiol 21:57-82.

Muller RU, Kubie JL, Bostock E, Taube JS, Quirk GJ (1991) Spatial firing correlates of neurons in the hippocampal formation of freely moving rats. In: Brain and space (Paillard J, ed), pp 296-333. Oxford, UK: Oxford UP. 
Neave N, Nagle S, Aggleton JP (1997) Evidence for the involvement of the mammillary bodies and cingulum bundle in allocentric spatial processing by rats. Eur J Neurosci 9:941-955.

O'Keefe J, Conway DH (1978) Hippocampal place units in the freely moving rat: why they fire where they fire. Exp Brain Res 31:573-590.

O'Keefe J, Dostrovsky J (1971) The hippocampus as a spatial map. Preliminary evidence from unit activity in the freely-moving rat. Brain Res 34:171-175.

O'Keefe J, Speakman A (1987) Single unit activity in the rat hippocampus during a spatial memory task. Exp Brain Res 68:1-27.

Paxinos G, Watson C (1986) The rat brain in stereotaxic coordinates. Sydney: Academic.

Quirk GJ, Muller RU, Kubie JL (1990) The firing of hippocampal place cells in the dark depends on the rat's recent experience. J Neurosci 10:2008-2017.

Redish AD, Touretzky DS (1997) Cognitive maps beyond the hippocampus. Hippocampus 7:15-35.

Redish AD, Touretzky DS (1999) Separating hippocampal maps. In: The hippocampal and parietal foundations of spatial cognition (Burgess N, Jeffrey KJ, O'Keefe J, eds), pp 204-219. Oxford: Oxford UP.

Samsonovich A, McNaughton BL (1997) Path integration and cognitive mapping in a continuous attractor neural network model. J Neurosci 17:5900-5920.

Save E (1997) The contribution of visual and inertial mechanisms to navigation in total darkness. Anim Learn Behav 25:324-334.

Save E, Cressant A, Thinus-Blanc C, Poucet B (1998) Spatial firing of hippocampal place cells in blind rats. J Neurosci 18:1818-1826.

Sharp PE, Kubie JL, Muller RU (1990) Firing properties of hippocampal neurons in a visually symmetrical environment: contributions of multiple sensory cues and mnemonic processes. J Neurosci 10:3093-3105.

Sharp PE, Blair HT, Etkin D, Tzanetos DB (1995) Influences of vestibular and visual motion information on the spatial firing patterns of hippocampal place cells. J Neurosci 15:173-189.

Skaggs WE, McNaughton BL, Gothard KM, Markus EJ (1993) An information theoretic approach to deciphering the hippocampal code. In: Advances in neural information processing systems, Vol 5 (Hanson S, Cowan J, Giles L, eds), pp 1030-1037. San Mateo, CA: Kaufman. Smith PF (1997) Vestibular-hippocampal interactions. Hippocampus $7: 465-471$.

Stackman RW, Taube JS (1997) Firing properties of head direction cells in the rat anterior thalamic nucleus: dependence on vestibular input. J Neurosci 17:4349-4358.

Sutherland RJ, Hoesing JM (1993) Posterior cingulate cortex and spatial memory: a microlimnology analysis. In: Neurobiology of cingulated cortex and limbic thalamus (Vogt BA, Gabriel M, eds), pp 461-477. Boston: Birkhauser.

Sutherland RJ, Whishaw IQ, Kolb B (1988) Contributions of cingulate cortex to two forms of spatial learning and memory. J Neurosci 8:1863-1872.

Taube JS (1998) Head direction cells and the neurophysiological basis for a sense of direction. Prog Neurobiol 55:225-256.

van Groen T, Wyss JM (1990a) Connections of the retrosplenial granular a cortex in the rat. J Comp Neurol 300:593-606.

van Groen T, Wyss JM (1990b) The postsubicular cortex in the rat: characterization of the fourth region of the subicular cortex and its connections. Brain Res 529:165-177.

van Groen T, Wyss JM (1992) Projections from the laterodorsal nucleus of the thalamus to the limbic and visual cortices in the rat. J Comp Neurol 324:427-448.

van Groen T, Wyss JM (1995) Projections from the anterodorsal and anteroventral nucleus of the thalamus to the limbic cortex in the rat. J Comp Neurol 358:584-604.

van Groen T, Vogt BA, Wyss JM (1993) Interconnections between the thalamus and retrosplenial cortex in the rodent brain. In: Neurobiology of cingulate cortex and limbic thalamus (Vogt BA, Gabriel M, eds), pp 124-150. Boston: Birkhauser.

Vogt BA, Miller MW (1983) Cortical connections between rat cingulate cortex and visual, motor and post-subicular cortices. J Comp Neurol 216:192-210.

Warburton EC, Aggleton JP, Muir JL (1998) Comparing the effects of selective cingulate cortex lesions and cingulum bundle lesions on water maze performance by rats. Eur J Neurosci 10:622-634.

Whishaw IQ, Gorny B (1999) Path integration absent in scent-tracking fimbria-fornix rats: evidence for hippocampal involvement in "sense of direction" and "sense of distance" using self-movement cues. J Neurosci 19:4662-4673.

Wyss JM, van Groen T (1992) Connections between the retrosplenial cortex and the hippocampal formation in the rat: a review. Hippocampus $2: 1-11$.

Zilles K, Wree A (1995) Cortex: areal and laminar structure. In: The rat nervous system (Paxinos G, ed), pp 649-685. San Diego: Academic. 